\title{
Mode competition in a system of two parametrically driven pendulums with nonlinear coupling
}

\author{
E.J. Banning ${ }^{1}$, J.P. van der Weele*, J.C. Ross ${ }^{2}$, M.M. Kettenis ${ }^{2}$ \\ Center for Theoretical Physics, University of Twente, P.O. Box 217, 7500 AE Enschede. The Netherlands
}

Received 21 Feburary 1997

\begin{abstract}
This paper is part three in a series on the dynamics of two coupled, parametrically driven pendulums. In the previous parts Banning and van der Weele (1995) and Banning et al. (1997) studied the case of linear coupling; the present paper deals with the changes brought on by the inclusion of a nonlinear (third-order) term in the coupling. Special attention will be given to the phenomenon of mode competition.

The nonlinear coupling is seen to introduce a new kind of threshold into the system, namely a lower limit to the frequency at which certain motions can exist. Another consequence is that the mode interaction between $1 \alpha$ and $2 \beta$ (two of the normal motions of the system) is less degenerate, causing the intermediary mixed motion known as MP to manifest itself more strongly.
\end{abstract}

Keywords: Mode competition; Parametric resonance; Center manifold reduction; Symmetry breaking; Pendulums

\section{Introduction}

\subsection{The system}

One phenomenon of particular interest involving coupled oscillators is that of mode competition, in which two or more 'normal modes' of a system compete and form new, mixed motions. Fine observations of mode competition were made by Ciliberto and Gollub in the so-called Faraday experiment $[3,4]$, where a bowl filled with fluid is subjected to a vertical harmonic forcing. Depending on the amplitude and

\footnotetext{
*Corresponding author.

${ }^{1}$ Current address: EPT-RF, Shell International Exploration and Production B.V.. Volmerlaan 8, $2280 \mathrm{AB}$ Rijswijk, The Netherlands.

${ }^{2}$ Current address: Institute for Theoretical Physics, University of Amsterdam, Valckenierstraat 65, 1018 XE Amsterdam, The Netherlands.
} 


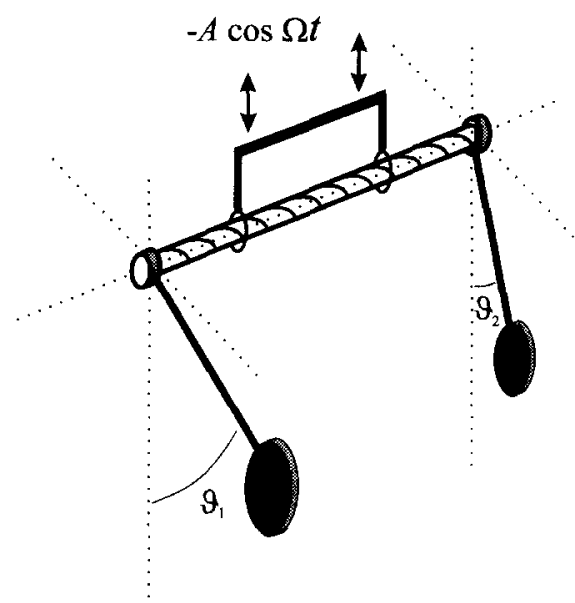

Fig. 1. The system, consisting of two parametrically driven pendulums, coupled by a nonlinear torsion spring. The length of the (identical) pendulums is taken to be $1 \mathrm{~m}$ and their mass $1 \mathrm{~kg}$.

frequency of the forcing, various wave patterns, or modes, become visible on the fluid surface. For certain values of the control parameters two of these modes are seen to compete, resulting in an alternation between the two modes which can be periodic, quasiperiodic and even chaotic. Similar instances of mode competition were reported by Lichter et al. in the behaviour of cross-waves in a long wave tank [5-7] and by Skeldon et al. in a perpendicular double pendulum [8,9]. In the present series of papers we describe mode competition in a particularly simple system, consisting of two identical pendulums coupled by a torsion spring, depicted in Fig. 1. The system is parametrically driven via the bar of suspension, which is being moved harmonically in the vertical direction with (angular) frequency $\Omega$ and amplitude $A$. The equations of motion are

$$
\begin{aligned}
& \ddot{\vartheta}_{1}+f(t) \sin \vartheta_{1}+\gamma \dot{\vartheta}_{1}+F\left(\vartheta_{1}, \vartheta_{2}\right)=0, \\
& \ddot{\vartheta}_{2}+f(t) \sin \vartheta_{2}+\gamma \dot{\vartheta}_{2}-F\left(\vartheta_{1}, \vartheta_{2}\right)=0,
\end{aligned}
$$

where $f(t)$ contains the driving force

$$
f(t)=\frac{1}{l}\left(g+A \Omega^{2} \cos \Omega t\right),
$$

the terms $\gamma \dot{\vartheta}_{1}$ and $\gamma \dot{\vartheta}_{2}$ represent viscous damping in the pivots, and the function $F\left(\vartheta_{1}, \vartheta_{2}\right)$ stands for the coupling between the pendulums. In Refs. [1,2] we treated the linearly coupled system, with $F\left(\vartheta_{1}, \vartheta_{2}\right)=K\left(\vartheta_{1}-\vartheta_{2}\right)$, and in the present paper $F$ is taken to be nonlinear:

$$
F\left(\vartheta_{1}, \vartheta_{2}\right)=K\left(\vartheta_{1}-\vartheta_{2}\right)+L\left(\vartheta_{1}-\vartheta_{2}\right)^{3} .
$$

Terms of even order are not included since these would give rise to a nonsymmetrical coupling; we choose to keep the problem symmetrical. The coefficients $K$ and $L$ will be 
taken to be positive. In the present context a negative $L$ is less realistic since in that case the pendulums would repel each other as soon as $\left|\vartheta_{1}-\vartheta_{2}\right|>\sqrt{K /(-L)}$; it is hard to imagine a torsion spring with this property. To facilitate comparison with our earlier results $[1,2]$ we take $K=1 \mathrm{~s}^{-2}$ in the numerical calculations. For the thirdorder term we sometimes use $L=0.01 \mathrm{~s}^{-2}$, if we want to illuminate the connection with linear coupling, but most of the calculations are performed with the relatively large value $L=0.2 \mathrm{~s}^{-2}$.

Eq. (1.1) describe the dynamics of the system in its five-dimensional phase space. We are primarily interested in motions which are periodic with period $T$ or $2 T$, where $T=2 \pi / \Omega$ is the period of the driving. It is therefore convenient to study the so-called stroboscopic map, obtained by sampling the pendulums' angles and angular velocities every time the bar of suspension reaches its lowest point. The periodic motions will then appear as fixed points in the four-dimensional stroboscopic phase space, and their stability is governed by the eigenvalues of the linearized stroboscopic map. These eigenvalues come either in complex conjugate pairs $(\lambda, \bar{\lambda})$ with $|\lambda|=e^{-n \gamma_{g} / 2}$, in real pairs $\left(\lambda, \lambda^{-1} e^{-n \gamma_{\Omega}}\right)$, or all together in a quadruplet $\left(\lambda, \bar{\lambda}, \lambda^{-1} e^{-n \gamma_{\Omega}}, \bar{\lambda}^{-1} e^{-n \gamma_{\Omega}}\right)$. Here $\gamma_{\Omega}=2 \pi \gamma / \Omega$ denotes the dimensionless 'effective' damping [2], and $n$ is an integer representing the periodicity of the motion with respect to the driving (i.e., $n=1$ or 2 in our case). When all four eigenvalues lie inside the unit circle the motion is stable, when one of the eigenvalues lies outside the unit circle the motion is called semi stable and, finally, when two of the eigenvalues lie outside the unit circle the motion is (totally) unstable. Note that a motion in a Hamiltonian system can at most be marginally stable (with all of its four eigenvalues on the unit circle), but for convenience we will usually leave out the word 'marginal'. That is, a centre in stroboscopic phase space will also be called stable.

The stability of a motion can change in one of three ways: by a period doubling bifurcation (where an eigenvalue passes -1 ), by a symmetry-breaking or equal-period bifurcation (where an eigenvalue passes +1 ), or by a Hopf bifurcation (in which two of the eigenvalues of a quadruplet cross the unit circle). Apart from these three types of bifurcation we will also encounter saddle-node bifurcations (with an eigenvalue at $+1)$.

\subsection{The symmetries and the motions}

It is evident from Fig. 1 and Eq. (1.1) that the system under consideration is highly symmetrical. The symmetries that are relevant in the context of our investigation are associated with reflection, exchange of pendulums, and time translation by one driving period (the equations of motion are equivariant with respect to these operations, see also Ref. [2]):
$\mathbf{R}:\left(\vartheta_{1}, \dot{\vartheta}_{1}, \vartheta_{2}, \dot{\vartheta}_{2}, t\right) \rightarrow\left(-\vartheta_{1},-\dot{\vartheta}_{1},-\vartheta_{2},-\dot{\vartheta}_{2}, t\right)$,
$\mathbf{E}:\left(\vartheta_{1}, \dot{\vartheta}_{1}, \vartheta_{2}, \dot{\vartheta}_{2}, t\right) \rightarrow\left(\vartheta_{2}, \dot{\vartheta}_{2}, \vartheta_{1}, \dot{\vartheta}_{1}, t\right)$,
$\mathbf{T}:\left(\vartheta_{1}, \dot{\vartheta}_{1}, \vartheta_{2}, \dot{\vartheta}_{2}, t\right) \rightarrow\left(\vartheta_{1}, \dot{\vartheta}_{1}, \vartheta_{2}, \dot{\vartheta}_{2}, t+2 \pi / \Omega\right)$. 


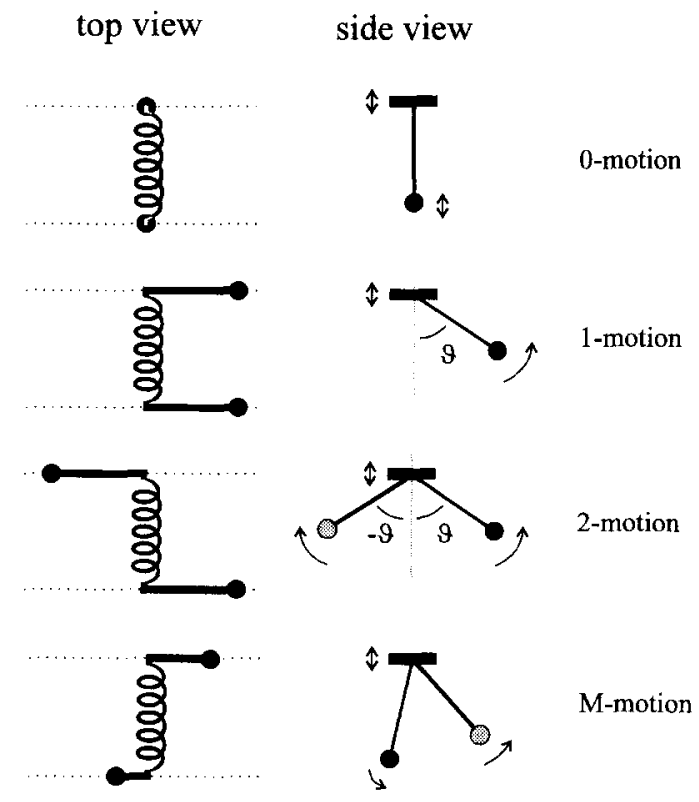

Fig. 2. The four types of motion.

Since these operations are their own inverse, ${ }^{2}$ we may write the symmetry group of the system as $\boldsymbol{Z}_{2}(\mathbf{R}) \times \boldsymbol{Z}_{2}(\mathbf{E}) \times \boldsymbol{Z}_{2}(\mathbf{T})$, which contains $2^{3}$ elements (including the identity transformation). In the Hamiltonian case $(\gamma=0)$ the symmetry of time reversal,

$$
\mathbf{t}:\left(\vartheta_{1}, \dot{\vartheta}_{1}, \vartheta_{2}, \dot{\vartheta}_{2}, t\right) \rightarrow\left(\vartheta_{1},-\dot{\vartheta}_{1}, \vartheta_{2},-\dot{\vartheta}_{2},-t\right),
$$

is also present and the symmetry group is extended to $\boldsymbol{Z}_{2}(\mathbf{R}) \times \boldsymbol{Z}_{2}(\mathbf{E}) \times \boldsymbol{Z}_{2}(\mathbf{T}) \times \boldsymbol{Z}_{2}(\mathbf{t})$, with $2^{4}$ elements.

The motions exhibited by the system can, but need not, possess (some of) the symmetries mentioned above. A motion is said to possess a symmetry if all the points on the phase-space trajectory of that motion are invariant under the corresponding transformation. The 0-motion (see Fig. 2), in which the pendulums simply move up and down with the driving, is the only motion which possesses all the symmetries of the system; in the present paper we find it convenient to regard it as a motion of period $2 T$. The 1-motions, in which both pendulums swing in phase, possess at least the E symmetry; the 2-motions, in which the pendulums are always in exact counterphase, possess at least the (combined) RE symmetry. The latter two categories are called normal motions because their trajectories in stroboscopic phase space are restricted to invariant planes, known as the 1-plane and the 2-plane, respectively. As a consequence,

\footnotetext{
${ }^{2}$ For the time-translation this is only true if we restrict ourselves to motions of period $2 T$; only then can $t=2 T$ be identified with $t=0$.
} 
their eigenvalues can be grouped into pairs (known as the 1- and 2-eigenvalues, corresponding to directions in and perpendicular to these planes, respectively $[1,2])$ which means that the normal motions can never undergo a Hopf bifurcation. The other motions considered in the present paper use all four dimensions of stroboscopic phase space and fall into the category of mixed motions, or M-motions. When dealing with the various motions we will indicate their symmetry properties, by which it is often possible to uniquely classify them.

The stability regions and bifurcations of the prominent motions play an important role in our analysis. Interestingly enough, the 0 -motion and the 1-motions are left unchanged by the inclusion of a third-order term in the coupling. The reason for this is simply that the spring is not stretched at all in these motions; the position of the fixed points can therefore not be a function of $K$ or $L$. Furthermore, their stability in the 2-direction is governed by the effect of infinitesimal changes around $\vartheta_{1}-\vartheta_{2}=0$, and only the linear coupling terms come into play here. Thus, for the 0 -motion and the 1 -motions we refer to our earlier work on linear coupling [1,2]. All other motions are affected by the third-order term, as we will see in the remainder of this paper. In Section 2 we will deal with them for the Hamiltonian case, and in Section 3 for the dissipative system. It turns out that the third-order term in the coupling removes a degeneracy of the linearly coupled system, making our results more generic and the similarities with other (experimental) systems more apparent. In the final section we summarize our main results and draw conclusions.

\section{The Hamiltonian case}

\subsection{Introduction}

In this section we explore the system defined by Eq. (1.1) for the Hamiltonian case, i.e., for $\gamma=0$. For the labeling of the various bifurcation lines (appearing as solid lines in the $(A, \Omega)$-plane) we will use a convention similar to that of Refs. [1,2]. A lowercase character thus symbolizes a period doubling or a saddle-node bifurcation, where two eigenvalues are at -1 . An uppercase character denotes a symmetry-breaking or a saddle-node bifurcation, with two eigenvalues at +1 . Hopf bifurcation lines form an exception to this rule, and will be indicated by the symbol $\mathbf{H}$. Lines labeled by the same character have unfolded from one $K=L=0$ line; indices are used in these cases to distinguish the various lines. We will frequently include lines which are meant as guide to the eye. Dotted lines in the stability diagrams then indicate the position of the bifurcation lines for linear coupling (i.e., for $L=0$ ) and dashed lines, labeled by open-faced characters, represent bifurcation lines not of the motion under consideration but of other motions.

As stated in the previous section we skip treatment of the 0-motion and the 1-motions; for these motions we refer to our earlier work [1,2]. In Section 2.2 we therefore immediately go to the $2 \alpha$-motion and the $2 \beta$-motion. The $2 \alpha$-motion is 
characterized by the fact that the pendulums reach their maximum angle the instant the bar of suspension goes through its lowest point; in the $2 \beta$-motion they reach their maximum angular velocity at this moment. It will be shown that the third-order term in the coupling introduces a frequency threshold for these motions. Above this threshold there is a hard and a soft version of each of them; below the threshold the 2-motions cannot exist. We then go on to the mixed motions, which are born from the 1- and 2-motions by bifurcations in directions out of their 'normal' planes. One of these mixed motions (type D) is born from $2 \alpha$ and will be treated in the same subsection as its mother orbit. The others are dealt with in Sections 2.3 and 2.4. In Section 2.3 we discuss type A and its bifurcation product B, and in Section 2.4 we deal with MP and ML. The latter two constitute the mode interaction in the Hamiltonian system: the MP-motion (Mixed Phase) connects the two normal motions $1 \alpha$ and $2 \beta$, and the ML-motion (Mixed Link) connects MP and A.

\subsection{The 2-motions and the mixed motion type $D$}

\subsubsection{The hard-soft transition}

Before we go into the details of the 2-motions, it is useful to consider some general features of an oscillator with a nonlinear restoring force. When the restoring force is sublinear, or 'softer than linear', the oscillator is said to be soft. In that case the period of the oscillation increases with its amplitude. When the restoring force is superlinear, or 'harder than linear', the oscillator is called hard. The period of oscillation then decreases with increasing amplitude. The intermediate case, an oscillator with a linear restoring force, is neither soft nor hard (it is harmonic) and the period of oscillation is constant.

In the present system there are two competing effects. The contribution to the restoring force from gravity is soft, whereas that from the coupling is hard. When the system is performing a 2 -motion (be it $2 \alpha$ or $2 \beta$ ) both these effects come into play, and the question arises whether this results in a soft 2 -motion (for which the period increases with its amplitude) or a hard one (for which the period decreases with its amplitude). The answer is that we find both. More specifically, at one and the same set of driving parameters $(A, \Omega)$ we may find both a soft 2-motion with a small amplitude, and a hard 2-motion with a larger amplitude. This is depicted in Fig. 3, where we have plotted the amplitude $\vartheta_{\max }$ of the $2 \alpha$-motion, for $L=0.01 \mathrm{~s}^{-2}$ (a rather low value of $L$ ), as a function of $(A, \Omega)$. The figure shows a sheet which is folded back; the lower part corresponds with the soft version of the $2 \alpha$-motion and the upper part (extending to infinite $\Omega$ ) with the hard version. At the folding line $\mathbf{X} \alpha$ the two versions are born simultaneously by means of a saddle-node bifurcation, and for frequencies to the left of this line the $2 \alpha$-motions do not exist. This means that the nonlinear coupling has introduced a frequency threshold into the system. Also for the $2 \beta$-motion we find similar behavior, with a frequency threshold(or saddle-node bifurcation line) $\mathbf{X} \beta$. We will repeatedly come across the thresholds in the next subsections; in the stability 


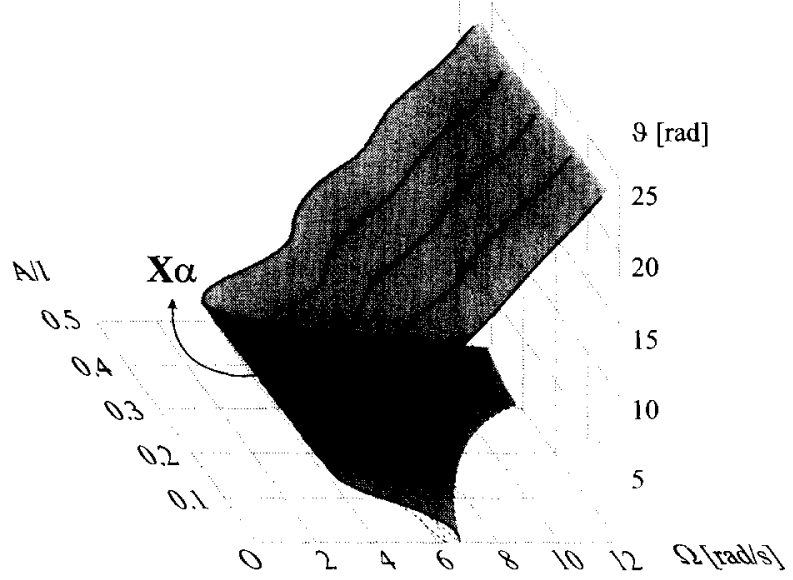

Fig. 3. Three-dimensional representation of the $2 \alpha$-motion. for $L=0.01 \mathrm{~s}^{-2}$. The lower and upper sheet represent the soft and hard versions, respectively.

diagrams they will be indicated by a curly hatch-pattern, implying the nonexistence of the motion in question on that side of the line.

\subsubsection{The $2 \alpha$-motion}

The symmetries of the $2 x$-motion are left unchanged by the inclusion of a thirdorder coupling term; that is to say, its symmetry group is given by $Z_{2}(\mathbf{R T}) \times Z_{2}(\mathbf{E T}) \times Z_{2}(\mathbf{t})$ which has the following eight elements: $\mathbf{R T}, \mathbf{E T}, \mathbf{t}, \mathbf{R E}, \mathbf{R T t}$, REt, ETt and 1. (Note that there is some freedom to choose the three generators of the $Z_{2} \times Z_{2} \times Z_{2}$ group; instead of RT, ET, $\mathbf{t}$ one might also choose for instance RT, RE, t or RT, RE, ETt.) A motion which possesses these (and only these) symmetries can immediately be classified as $2 \alpha$. In Fig. 4 a we show the stability diagram of the soft version of the $2 \alpha$-motion, for $L=0.01 \mathrm{~s}^{-2}$. As discussed above, at the frequency threshold line $\mathbf{X} \alpha$ (which intersects the $\Omega$-axis at the critical frequency $\Omega_{\mathrm{c}}$ ) the soft and hard version of $2 \alpha$ are born simultaneously in a saddle-node bifurcation.

Let us look at the bifurcational life of the soft $2 \alpha$-motion in some more detail. At line $\mathbf{A}_{\mathbf{1}}$ it is born from the 0-motion in a period doubling bifurcation; the 2-eigenvalues are at +1 along this line, while the 1-eigenvalues lie on the unit circle (see also Ref. [1,2]). Traversing the $(A, \Omega)$-plane from right to left the 1-eigenvalues travel around the unit circle (the symmetry of the $2 \alpha$-motion prohibits them from entering the negative real axis $[1,10])$, to finally enter the positive real axis at line $\mathbf{D}_{\mathbf{I}}$. Here the soft $2 \alpha$-motion becomes semi stable and gives birth to a mixed motion of type $\mathrm{D}$. We shall come back to type $\mathrm{D}$ at the end of this subsection. The 2-eigenvalues follow the 1-eigenvalues in their journey. For sufficiently high values of the driving amplitude $A$ they manage to reach +1 and enter the positive real axis at line $\mathbf{D}_{\mathrm{II}}$, to subsequently turn in their 


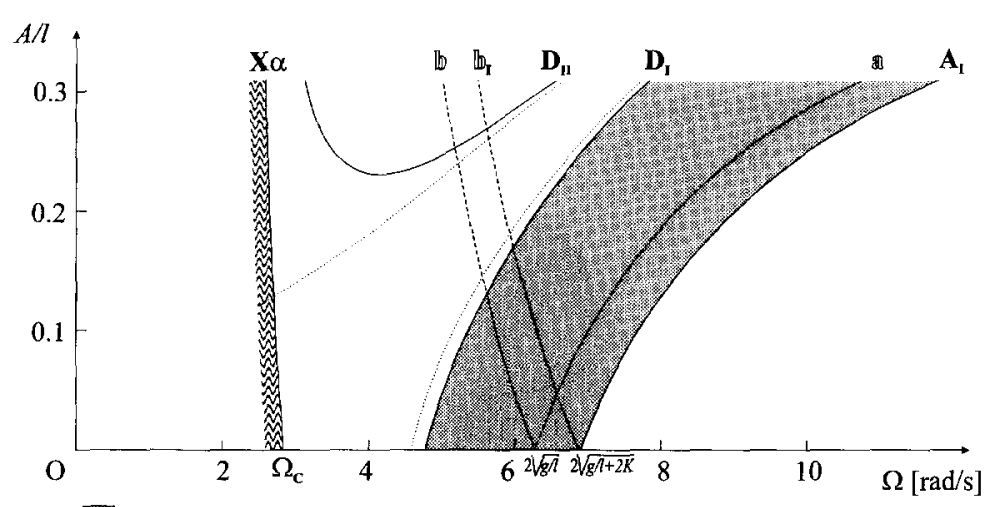

(a) Stable (soft) $2 \alpha$-motion (for $K=1 \mathrm{~s}^{-2}, L=0.01 \mathrm{~s}^{-2}$ )

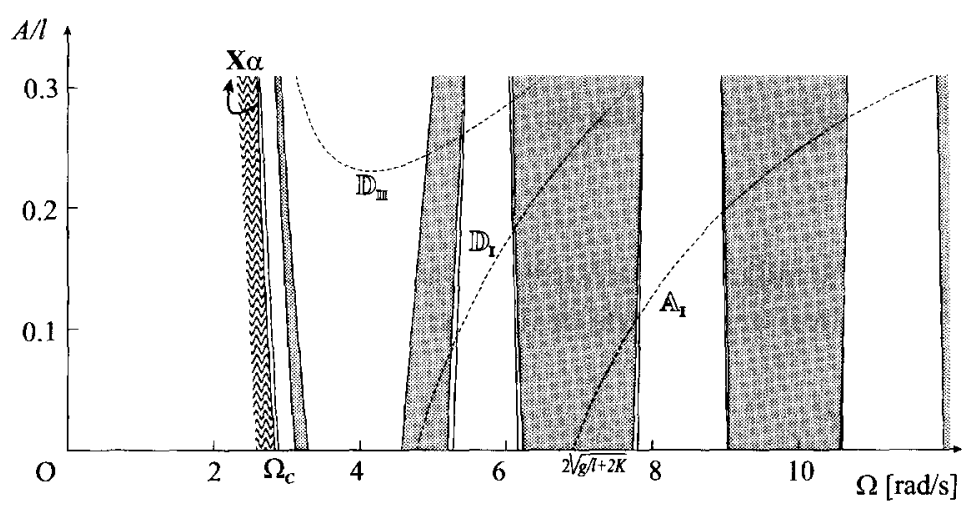

(b) Stable (hard) $2 \alpha$-motion (for $K=1 \mathrm{~s}^{-2}, L=0.01 \mathrm{~s}^{-2}$ )

Fig. 4. (a) Stability diagram of the soft variation of the $2 \alpha$-motion, for $L=0.01 \mathrm{~s}^{-2}$. The dotted lines represent the case of linear coupling $(L=0)$. (b) Stability diagram of the hard variation of the $2 \alpha$-motion, for $L=0.01 \mathrm{~s}^{-2}$. The dashed lines represent bifurcation lines of the soft $2 \alpha$-motion.

tracks and reenter the unit circle again at (the left part of) line $\mathbf{D}_{\text {II }}$. For smaller values of $A$ the 2-eigenvalues never reach +1 , but reverse direction at some point on the unit circle. In either case the 2-eigenvalues travel back around the unit circle to return to +1 at line $\mathbf{X} \alpha$, where the semi stable soft $2 \alpha$-motion and its totally unstable hard counterpart annihilate each other in a saddle-node bifurcation. The amplitude of the motion is quite large here, with pendulums swinging up higher than $\pi$ radians.

We now turn to the hard version. In Fig. 4b, looking with increasing drive frequency (as is appropriate for hard oscillations) we see its creation at line $\mathbf{X} \alpha$, with its 1-eigenvalues on the positive real axis at a large distance from +1 . The 2-eigenvalues (starting at +1 ) make a short excursion on the positive real axis but soon rejoin the unit circle; the hard $2 \alpha$-motion then becomes semi stable. Soon thereafter the 1 eigenvalues enter the unit circle too and the motion becomes fully stable. They do not 
wander far from +1 but, upon further increasing $\Omega$, reverse direction on the unit circle and reenter the positive real axis, rendering the motion semi stable once more.

The hard $2 \alpha$-motion does not become stable just once. Continuing to the right in the $(A, \Omega)$-plane, we see the motion losing and regaining stability again and again. The recurrent stability is related to the fact that the amplitude of the motion grows indefinitely (with increasing $\Omega$ ) and that the gravitational part of the restoring force is a periodic function of $\vartheta$. The hard $2 x$-motion is stable for the first time when the amplitude of the pendulums is in a (small) interval somewhere between $\pi$ and $3 \pi$; it is stable for the second time in an amplitude interval between $3 \pi$ and $5 \pi$; for the third time between $5 \pi$ and $7 \pi$, and so on. The corresponding stable zones in the $(A, \Omega)$-plane become wider and wider. This does not mean, however, that they also become more important. One should realize that the stable zones at higher frequencies correspond to exceptionally violent motions. In the third stable region in Fig. $4 b$ for instance, the pendulums would be rotating almost seven times in one direction, then almost seven times in the other direction, all within two drive periods (about half a second at this point). In a practical realization of our system such motions can hardly be expected to be stable, and indeed their stability here is a consequence of the fact that, up to now, we have neglected the influence of dissipation. In practice, some dissipation will always be present, and this will particularly affect the more violent motions. In Section 3 we will see that the stable zones disappear from the picture as soon as we introduce dissipation into the system.

We now go on to study a larger value of $L$, namely $L=0.2 \mathrm{~s}^{-2}$. The stability diagrams for the soft and hard variations are depicted in Fig. 5a and Fig. 5b. The most important observation to be made from these diagrams is that the saddle-node line $\mathbf{X} x$ now lies well within the region of instability of the 0 -motion. From a first glance at Fig. 5 a one might be tempted to think that line $\mathbf{D}_{\mathbf{l}}$, where the mixed motion of type D is born, has been cut off prematurely by $\mathbf{X} x$. However, in Fig. $5 b$ (note the different frequency scale) we see that this is actually not the case: the lower part of $\mathbf{D}_{\mathbf{I}}$ is folded back at the point where it touches $\mathbf{X} x$. Indeed, the upper part of line $\mathbf{D}_{\mathbf{1}}$ is a bifurcation line of the soft $2 x$-motion, while the lower part belongs to the hard version.

From Fig. $5 \mathrm{a}$ we see that the soft $2 x$-motion is born completely stable from the 0 -motion at line $\mathbf{A}_{\mathbf{I}}$; its region of stability is bounded by lines $\mathbf{A}_{\mathbf{I}}$ and $\mathbf{D}_{\mathbf{I}}$ for high values of the driving amplitude $A$, and by lines $\mathbf{A}_{\mathbf{1}}$ and $\mathbf{X} x$ for low values. So, for high $A$ it arrives at the line $\mathbf{X} \alpha$ semi stable and recombines with an unstable hard $2 \alpha$-motion (Fig. 5b). For low values of $A$ the soft motion arrives at the recombination line $\mathbf{X} x$ fully stable; here the hard motion is born semi stable.

The diagrams in Fig. 5a and Fig. 5b and Fig. 4a and Fig. 4b are quite alike from a qualitative point of view, even though the quantitative differences are huge. For example, for the soft version line $\mathbf{D}_{\mathrm{II}}$ still exists (between $\mathbf{X} \alpha$ and $\mathbf{D}_{\mathrm{I}}$ ) but it has been shifted to the higher regions of the $(A, \Omega)$-plane. For the hard motion the recurrent stable zones have widened significantly, and have been shifted to the right. As discussed before, however, they are of little practical significance. 


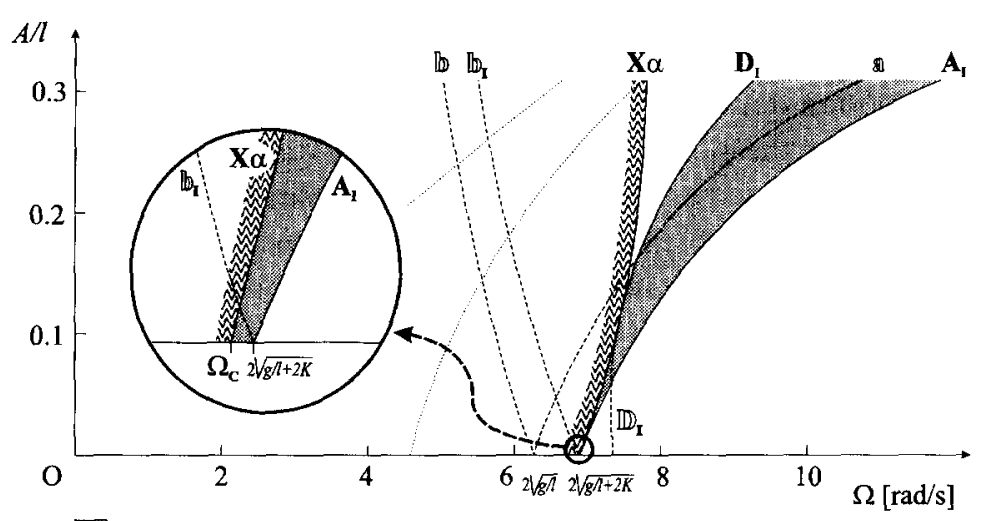

(a) Stable (soft) $2 \alpha$-motion (for $K=1 \mathrm{~s}^{-2}, L=0.2 \mathrm{~s}^{-2}$ )

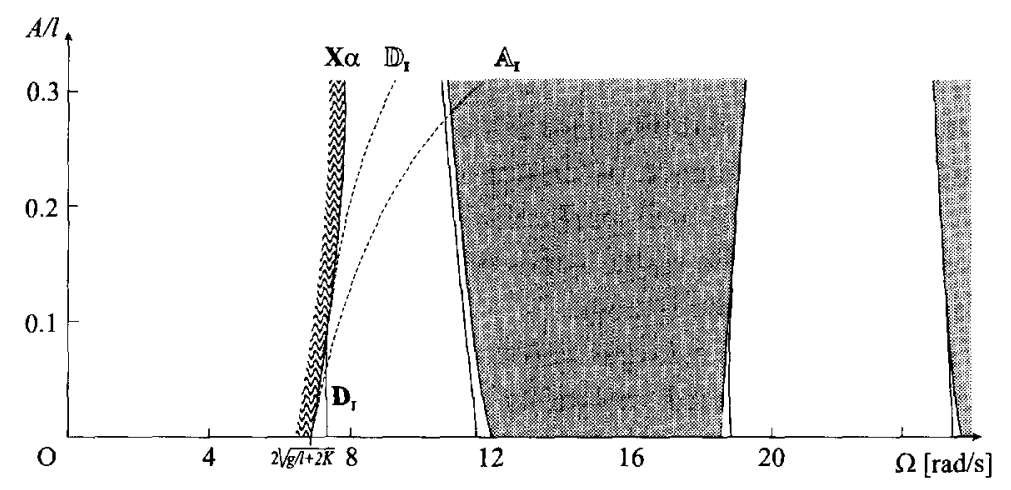

(b) Stable (hard) $2 \alpha$-motion (for $K=1 \mathrm{~s}^{-2}, L=0.2 \mathrm{~s}^{-2}$ )

Fig. 5. (a) Stability diagram of the soft variation of the $2 \alpha$-motion, for $L=0.2 \mathrm{~s}^{-2}$. The dashed lines represent bifurcation curves of the 0 -motion. (b) Stability diagram of the hard variation of the $2 \alpha$-motion, for $L=0.2 \mathrm{~s}^{-2}$. The dashed lines represent bifurcation curves of the soft $2 \alpha$-motion. Note the different frequency scale.

\subsubsection{The $2 \beta$-motion}

The qualitative difference between $2 \alpha$ and $2 \beta$ is, as mentioned earlier, the phase relation of the pendulums with respect to the driving: for $2 \alpha$ the pendulums reach their maximum deflection at the moment of stroboscopic sampling, whereas for $2 \beta$ they reach their maximum angular velocity. The symmetries of $2 \beta$ are therefore very much like those of $2 \alpha$; the only difference is that every occurrence of the timereversal symmetry $\mathbf{t}$ is now replaced by Tt. Thus, its symmetry group is $Z_{2}(\mathbf{R T}) \times Z_{2}(\mathrm{ET}) \times Z_{2}(\mathrm{Tt})$, with elements RT, ET, Tt, RE, Rt, RETt, Et and 1. Just as for the $2 \alpha$-motion there exist a soft and a hard variation of $2 \beta$; their stability diagrams (for $L=0.01 \mathrm{~s}^{-2}$ ) are depicted in Fig. 6a and Fig. 6b. Going from left to right in these diagrams the two variations are created simultaneously in a saddle-node bifurcation 


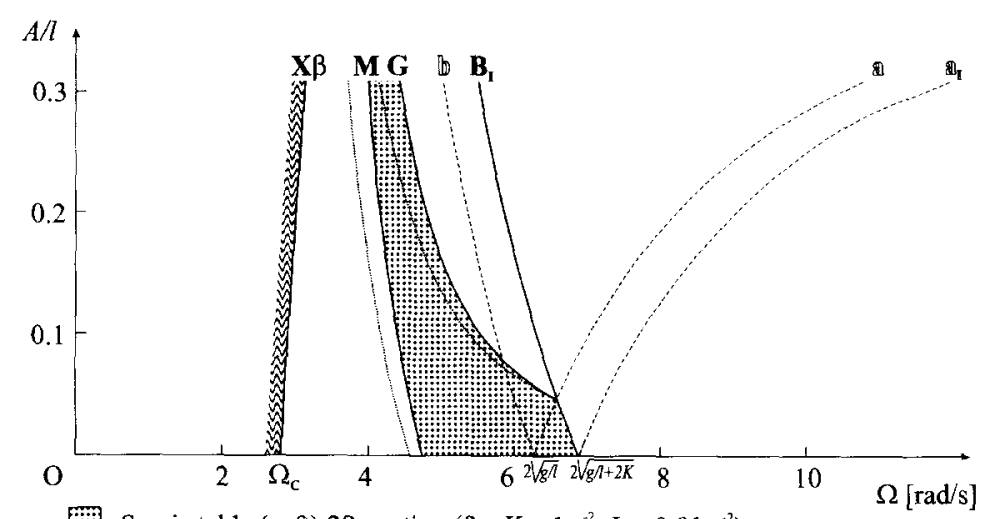

(a) Semi stable (soft) $2 \beta$-motion (for $K=1 \mathrm{~s}^{-2}, L=0.01 \mathrm{~s}^{-2}$ )

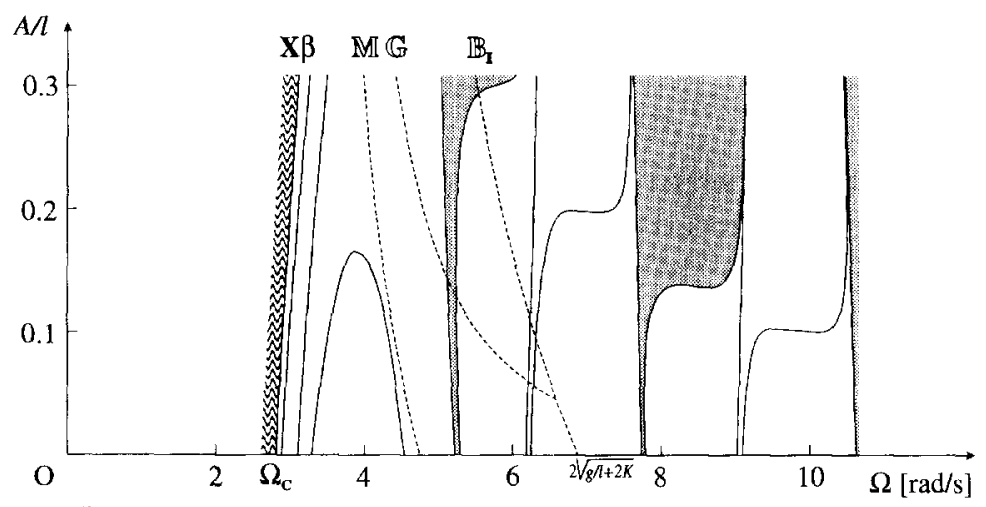

(b) Stable (hard) $2 \beta$-motion (for $K=1 \mathrm{~s}^{-2}, L=0.01 \mathrm{~s}^{-2}$ )

Fig. 6. (a) Stability diagram of the soft variation of the $2 \beta$-motion, for $L=0.01 \mathrm{~s}^{-2}$. The dotted lines represent bifurcation curves of the 0 -motion. (b) Stability diagram of the hard variation of the $2 \beta$-motion, for $L=0.01 \mathrm{~s}^{-2}$. The dashed lines represent bifurcation curves of the $2 \beta$-motion.

at line $\mathbf{X} \beta$. Since $2 \alpha$ and $2 \beta$ are indistinguishable when driving is absent, lines $\mathbf{X} \beta$ and $\mathbf{X} \propto$ must reach the $\Omega$-axis at the same value of $\Omega$. This holds, in fact, for all bifurcation curves of the $\alpha$ and $\beta$ variations.

The soft variation of $2 \beta$ is born from the 0 -motion at line $\mathbf{B}_{\mathbf{1}}$, by means of a period doubling bifurcation in the 2-direction. Going from right to left in the $(A, \Omega)$-plane the 2 -eigenvalues of the $2 \beta$ motion travel away from +1 on the positive real axis. Not before long, though, they turn back and reach +1 again at line $\mathbf{X} \beta$. In the meantime, the 1-eigenvalues are on the positive real axis, except in the region between lines $\mathbf{G}$ and $\mathbf{M}$, where they make a trip around the unit circle. At the saddle-node line $\mathbf{X} \beta$ the 1-eigenvalues are on the positive real axis, a long distance from +1 .

The hard variation of $2 \beta$ (see Fig. $6 \mathrm{~b}$ ) is thus born semi stable along line $\mathbf{X} \beta$, with its 1-eigenvalues on the positive real axis and the 2-eigenvalues starting their trip around 


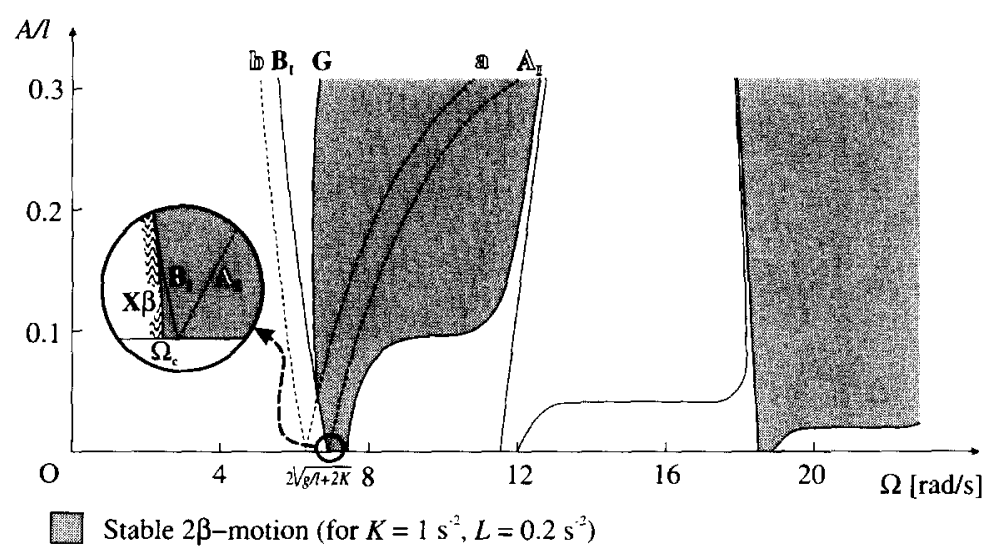

Fig. 7. Stability diagram of the $2 \beta$-motion, for $L=0.2 \mathrm{~s}^{-2}$. There is only a hard version for this value of $L$, except in the tiny region between lines $\mathbf{B}_{1}$ and $\mathbf{X} \beta$ (see enlargement) where both a stable hard and a semi stable soft version of the $2 \beta$-motion exist.

the unit circle. Following events from left to right in the $(A, \Omega)$-plane, as is appropriate for a hard motion, we find recurrent stable zones which become more prominent as we go to the right, much like in the case of the hard $2 \alpha$-motion. This was to be expected, since every bifurcation line (emanating from the $\Omega$-axis) of the hard $2 \alpha$-motion must have its counterpart for the hard $2 \beta$-motion, as explained above. In this context, it may also be noted that the stability with respect to the 2 -direction of $2 \beta$ near the $\Omega$-axis is complementary to that of $2 \alpha$.

Let us now consider a large value of $L$. The situation for $L=0.2 \mathrm{~s}^{-2}$ is depicted in Fig. 7. For this value the line $\mathbf{X} \beta$ has been pushed so far to the right that it has, for the better part, melted together with line $\mathbf{B}_{\mathrm{I}}$, causing the period doubling bifurcation of the 0-motion to switch from super- to subcritical. That is, everywhere along line $\mathbf{B}_{\mathrm{I}}$, except for a small part close to the $\Omega$-axis, the $2 \beta$-motion is born as a hard variation straightaway (see also Appendix B). The small part close to the $\Omega$-axis has to be there, since the $2 \alpha$-motion (see Fig. 5a) starts its life soft ${ }^{3}$ for this value of $L$ and the two motions are indistinguishable for $A=0$. So all that remains of the semi stable soft region is a tiny area between $\mathbf{B}_{\mathrm{I}}$ and what is left of $\mathbf{X} \beta$.

At line $\mathbf{B}_{\mathrm{I}}$ the hard $2 \beta$-motion is born either stable or semi stable, depending on whether we start below or above the intersection point of the two resonance tongues (which we shall frequently refer to as the primary mode interaction point). Line $\mathbf{G}$,

\footnotetext{
${ }^{3}$ Sufficiently close to the $\Omega$-axis (where we may ignore the driving term) the restoring force for the 2-motions in terms of the normal coordinate $\vartheta_{1}-\vartheta_{2}$ grows softer than linear for $L=0.2 \mathrm{~s}^{-2}$. But it is a borderline case, since the restoring force would become hardening for $L>g / 48 I=0.2041 \mathrm{~s}^{-2}$. (The same value of $L$ will be seen in Appendix B to mark the transition from the mode interaction scenario depicted in Fig. B.3 to the one shown in Fig. B.4.).
} 
where the 1 -eigenvalues of the $2 \beta$-motion are at +1 , acts as the borderline between the two regimes. This line has, following the trend set by $\mathbf{X} \beta$, been swept to the right by the action of the third-order coupling term (see Fig. 6a and 7); this has profound consequences for the mode interaction in the system, as we will demonstrate later on. One may further note that the stable region of $2 \beta$ is quite large; it totally engulfs the soft $2 x$-motion's stability zone (for reasonable values of $A$ ).

\subsubsection{Type $D$}

The mixed motion known as type $\mathrm{D}$ is the first motion type we come across which uses all four dimensions of stroboscopic phase space (a Hopf bifurcation is therefore now a distinct possibility). It is born in a symmetry-breaking bifurcation of the $2 x$-motion (be it soft or hard) in the 1-direction along line $\mathbf{D}_{\mathbf{l}}$. Type D may, even though we cannot really use linear superposition in our system, be described as a $2 x$-motion around a displaced downward equilibrium, i.e., the pendulum motions are centered around some nonzero angle (cf. Appendix A). As $\Omega$ is decreased we see this angle increase, whereas the amplitude of both pendulums around this angle hardly changes.

The symmetry group of type $\mathrm{D}$ is $Z_{2}(\mathrm{ET}) \times Z_{2}(\mathbf{t})$, with elements ET. ETt. $\mathbf{t}$ and 1. Since the group of its mother (the $2 \alpha$-motion) is $Z_{2}(\mathbf{R T}) \times Z_{2}(\mathbf{E T}) \times Z_{2}(\mathbf{t})$, one might be tempted to think that the RT symmetry has been broken in this bifurcation. However, since we have some freedom in our choice of group generators, we can also write the symmetry group of type $\mathrm{D}$ as $Z_{2}(\mathbf{E T}) \times Z_{2}(\mathbf{E T t})$ and that of $2 x$ as $Z_{2}(\mathbf{E T}) \times Z_{2}(\mathbf{E T t}) \times Z_{2}(\mathbf{R T t})$, so we may equally well say that it is the $\mathbf{R T t}$ symmetry that has been broken. The third and last way to write the symmetry group of type $D$ is $Z_{2}(\mathbf{E T t}) \times Z_{2}(\mathbf{t})$ and according to this choice the broken symmetry would be REt Indeed, as noted also in Ref. [2], the only unambiguous way to characterize a symmetry breaking is by summing up all symmetry elements lost in the bifurcation; in this case these elements are RT, RE, RTt and REt. In a sense, it would be more appropriate to speak of symmetry reduction rather than symmetry breaking.

The mixed motion of type $\mathrm{D}$ has a relatively simple stability diagram which. for $L=0.2 \mathrm{~s}^{-2}$, is presented in Fig. 8. Along the upper part of line $\mathbf{D}_{1}$, i.e. above the point where it touches $\mathbf{X} \alpha$, type D is born stable from the soft $2 \alpha$-motion. Its stability is rather short-lived though, since the eigenvalue pairs soon meet on the unit circle and undergo a Krein collision $[1,11,12]$ to form a quadruplet; the D-motion thus becomes (fully) unstable by means of a Hopf-bifurcation. Along the lower part of line $D_{1}$ the $\mathrm{D}$-motion is born from the hard $2 \alpha$-motion, when the 2-eigenvalues of the latter are on the positive real axis (and the 1-eigenvalues at +1 ). Also here the D-motion is born soft, i.e., towards the left in the $(A, \Omega)$-plane. Its eigenvalues travel towards each other on the positive real axis and at the moment of collision go off the axis into the complex plane. So, again we get a quadruplet; this time, however, not as the result of a Hopf bifurcation. In both cases (above and below the point where $\mathbf{D}_{1}$ touches $\mathbf{X} \alpha$ ) the quadruplet subsequently travels through the complex plane and eventually falls apart on the positive real axis; one may note that the negative real axis, due to the symmetry 


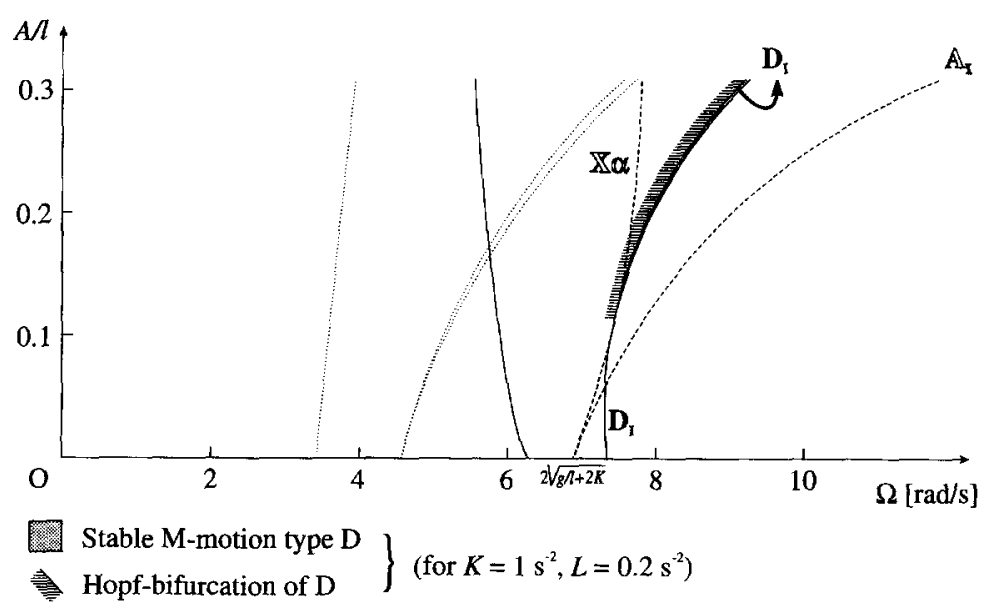

Fig. 8. Stability diagram of the mixed motion type $\mathrm{D}$, for $L=0.2 \mathrm{~s}^{-2}$. The dashed lines represent bifurcation lines of the $2 \alpha$-motion.

of type D, was not an option $[1,10]$. Finally, one of the eigenvalue pairs eventually enters the unit circle, rendering type D semi stable. Full stability, however, is never regained.

\subsection{Mixed motion types $A$ and $B$}

The mixed motion of type $\mathrm{A}$ is a bifurcation product of the $1 \alpha$-motion, resulting from a symmetry-breaking in the 2-direction. It can be viewed (see also Appendix A) as a $1 \alpha$-motion with a 2 -component superimposed on it, i.e., both pendulums move in phase with each other but with different amplitudes. The A-motion is the most prominent mixed motion of our system, with a large stable region in the center of the $(A, \Omega)$-plane. Its stability diagram is depicted in Fig. 9, for $L=0.2 \mathrm{~s}^{-2}$; in addition, the figure also shows data of one of A's bifurcation products, known as type B [1,2].

The $1 \alpha$-motion gives birth to type $\mathrm{A}$ in a symmetry-breaking bifurcation at line $\mathbf{A}_{\mathrm{II}}$. Type A has $Z_{2}(\mathbf{R T}) \times Z_{2}(\mathbf{t})$ as its symmetry group, with elements $\mathbf{R T}, \mathbf{t}$ and $\mathbf{R T t}$ and $\mathbf{1}$; that is, the symmetries which are lost in the bifurcation are E, Et, RETt, and RET. Along the upper part of its birthline two of the eigenvalues of type A reside on the positive real axis (while the other two are at +1 , of course). Decreasing $\Omega$, these eigenvalues approach the unit circle and enter it at line $\mathbf{B}_{\mathrm{II}}$. Thus, type $\mathrm{A}$ becomes stable by means of a symmetry-breaking bifurcation, giving birth to yet another mixed motion which we shall call Mixed Link (or ML), since it provides a link between type A and the Mixed Phase motion (MP), as discussed in Section 2.4. The bifurcation at line $\mathbf{B}_{\mathrm{II}}$ is characterized by the loss of $\mathbf{t}$ and $\mathbf{R T t}$ symmetry; ML is left with only $\mathbf{R T}$ and $\mathbf{1}$. 


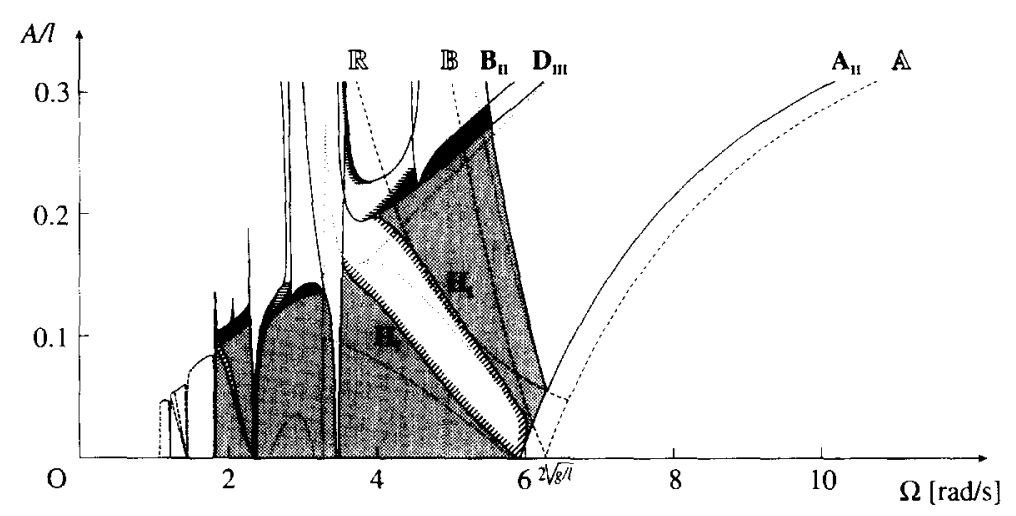

$\left.\begin{array}{l}\text { Stable M-motion type A } \\ \text { Hopf-bifurcation of A } \\ \text { Stable M-motion type B } \\ \text { Hopf-bifurcation of B }\end{array}\right\}$ (for $K=1 \mathrm{~s}^{2}, L=0.2 \mathrm{~s}^{2}$ )

Fig. 9. Stability diagram of the mixed motion type A, for $L=0.2 \mathrm{~s}^{-2}$. The dashed lines represent bifurcation curves of the $1 \alpha$-motion.

Looking at Fig. 9 we see that the stable region of the A-motion consists of many disconnected parts, or 'bubbles'. For linear coupling only two bubbles were discernible, i.e., the primary stable region (interrupted by $\mathbf{H}_{1}$ ) and the second one; the inclusion of the third-order term has caused the bubbles to become much more pronounced, and we can now distinguish a whole series of them. The gaps between them are related to the higher-order resonance tongues of the 0-motion (see also Ref. [1]). On top of the bubbles type $\mathrm{A}$ undergoes a symmetry-breaking bifurcation and gives birth to various mixed motions with only the $\mathbf{t}$ symmetry (and the identity transformation) left, collectively denoted as type B. We have seen a B-motion already in Ref. [1], namely the one that caps the primary bubble. It loses its stability by means of either a Hopf bifurcation or a period doubling. The stable B-regions on top of the higher-order bubbles were not discernible before and have come out as a result of the third-order coupling. It should be noted that the rightmost bifurcation line on top of the secondary bubble is not a bifurcation line of A, but is instead associated with a saddle-node bifurcation in which two B-motions with $\mathbf{t}$ symmetry are born. Indeed, this saddle-node line marks the point on the border of the second bubble where the bifurcation of A to B switches from super- to subcritical; on the lower right side an unstable B-motion is thus born towards the inside of the bubble.

At the left-hand side of the primary bubble (and of the third, fifth, seventh bubble and so on) type A gives way to a second variation of the B-motion, via yet another symmetry-breaking bifurcation. This type of B-motion cannot be connected to the first type, since it has RTt symmetry instead of $\mathbf{t}$; for the sake of simplicity we have omitted the bifurcation curves of this type. Indeed, a lot more could be said about the 
B-motions in general, but we shall not go into further details here. We will come back to them in Section 3, when we add dissipation to the system; the t-symmetry and all its combinations are then lost, causing the two kinds of B-motion to fall in the same symmetry class and thus enabling them to connect with each other.

Also the interior of the bubbles displays a striking reoccurring pattern. The odd-numbered bubbles are eroded from within, while the even-numbered bubbles are not. This is due to the fact that the eigenvalue pairs in the odd-numbered bubbles have opposite Krein signature, enabling them to form a quadruplet when they collide and render the A-motion unstable by means of a Hopf bifurcation. In the even-numbered bubbles the Krein signatures are identical, and Hopf bifurcations cannot occur. We refer the reader to Ref. [1], where we have discussed this in detail for the case of linear coupling; the third-order coupling term has made the pattern even more conspicuous without however bringing about any qualitative changes.

\subsection{The Mixed Phase and Mixed Link motions}

The Mixed Phase motion, or MP for short, is born from $1 \alpha$ along line $\mathbf{R}$ and from $2 \beta$ along line $\mathbf{G}$. Both birthlines emerge from the same point in the $(A, \Omega)$-plane, the primary mode interaction point, where $1 \alpha$ and $2 \beta$ are born simultaneously from the 0 -motion; MP forms, in fact, a link between these two 'normal modes'. We may view MP as a $2 \beta$-motion with an angular component in the 1-direction added to it, or equivalently, as a $1 \alpha$-motion with a superimposed velocity component in the 2 direction (see also Appendix A). In a previous paper [2] we commented upon the importance of the MP-motion for the mode interaction between $1 \alpha$ and $2 \beta$. In the case of linear coupling, however, this importance was mainly theoretical since the MPmotion existed only in a very thin region in the $(A, \Omega)$-plane. This can be attributed to the fact that the bifurcation problem close to the primary mode interaction point is (very nearly) degenerate in the linearly coupled case, as demonstrated in Appendix B. This means that lines $\mathbf{R}$ and $\mathbf{G}$ emerge from the primary mode interaction point in (almost exactly) the same direction. But in the present case the third-order coupling term has swept line $\mathbf{G}$ to the right (as noted already in connection with the $2 \beta$-motion) while, of course, line $\mathbf{R}$ has not been affected.

The stability diagram of MP is presented in Fig. 10, for $L=0.2 \mathrm{~s}^{-2}$. We see MP being born from the $1 \alpha$-motion in crossing line $\mathbf{R}$ from below. The symmetry group of MP is $Z_{2}(\mathbf{R T}) \times Z_{2}(\mathbf{E t})$, so the bifurcation is characterized by the loss of the elements E, RTt, RET and t. Since MP inherits the stability properties of $1 \alpha$, it is born semi stable along the left part of line $\mathbf{R}$ and stable along the right part. (The borderline between the semi stable and stable region is dubbed line $\mathbf{J}$; we will come to this in a moment.) After stabilization the MP-motion soon becomes unstable when its eigenvalues collide and form a quadruplet. Generically, the associated Hopf bifurcation line extends all the way to the primary mode interaction point (as can be argued using Krein theory [11,1]); all numerical evidence suggests that this is indeed the case. 


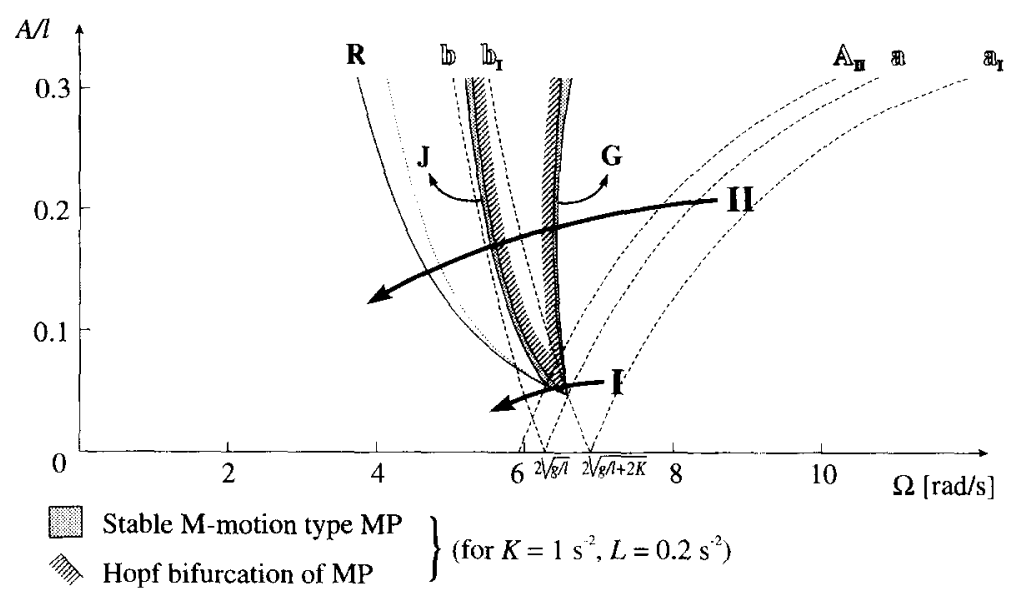

Fig. 10. Stability diagram for the MP-motion. for $L=0.2 \mathrm{~s}^{-2}$. The dashed lines represent bifurcation curves of type $\mathrm{A}$ and of the 0 -motion.

On the other side MP is born from the (hard) $2 \beta$-motion in a symmetry breaking bifurcation along line $\mathbf{G}$; in this bifurcation the elements Tt, RE, Rt and ET are lost. At line $\mathbf{G}$ the stability is passed on from $2 \beta$ to MP. Soon thereafter MP becomes (fully) unstable by means of a Hopf bifurcation, after which the motion goes on to meet $1 x$ at line $\mathbf{R}$. Along the way MP has to undergo one or more additional bifurcations, since it has to arrive at line $\mathbf{R}$ either semi stable (along the left part of $\mathbf{R}$ ) or completely stable (along the right part). Close to the mode interaction point this is accomplished by a second Hopf bifurcation (as already mentioned) and further away from this point it takes, apart from the Hopf bifurcation, yet another symmetry breaking, as can be seen in Fig. 11a and $11 \mathrm{~b}$. In these pictures we have schematically drawn the bifurcational structure along the arrows marked I and II in Fig. 10. Together they sum up many of the results of this section, and display how the mode interaction (between $1 \alpha$ and $2 \beta$ ) takes place in the Hamiltonian case.

There is one thing we have yet to discuss and that is line $\mathbf{J}$, where (if one travels along arrow II in Fig. 10) MP gains stability by means of a symmetry-breaking bifurcation, giving birth to the motion called Mixed Link, or ML for short. The symmetries lost in the bifurcation are those which contain time-reversal, i.e., RTEt and Et, leaving ML with only one nontrivial symmetry: RT. This agrees with what we found already in Section 2.3 where we also encountered ML, but then as a bifurcation product of the A-motion (along line $\mathbf{B}_{11}$ ). Indeed, the ML-motion connects MP and A. just as the MP-motion connects $1 \alpha$ and $2 \beta$. To make the analogy even stronger, lines $\mathbf{J}$ and $\mathbf{B}_{I I}$ emanate from one point, the secondary mode interaction point, just as lines $\mathbf{R}$ and $\mathbf{G}$ emanated from the primary mode interaction point. The common characteristic of these mode interaction points is that each of them coincides with the intersection of two bifurcation lines of one single motion; the primary mode interaction point lies at the intersection of the 0-motion's bifurcation lines a and $\mathbf{b}_{\mathbf{1}}$, and the secondary 


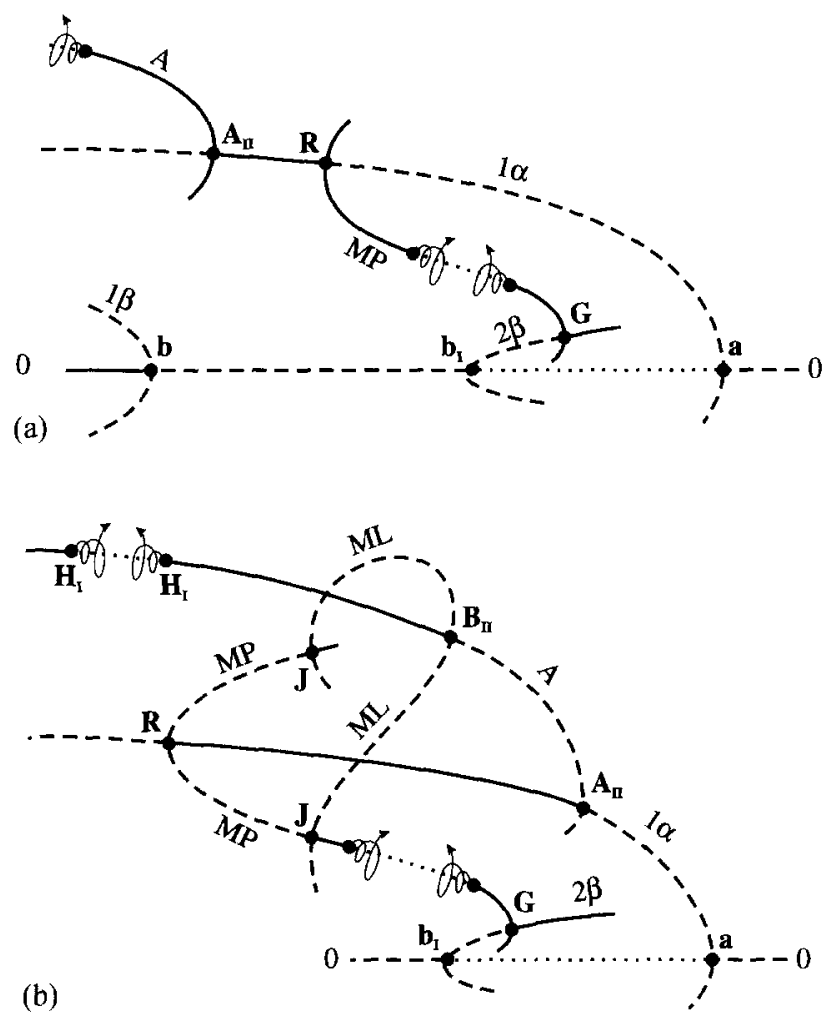

Fig. 11. (a) and (b) Schematic representation of the bifurcational structure along the arrows marked I and II in Fig. 10. In this figure and in all bifurcation diagrams to follow, stable solutions are represented by solid lines, semi stable solutions by dashed lines, and totally unstable solutions by dotted lines. Bifurcations are indicated by black bullets and Hopf bifurcations have, in addition to this, a spiraled arrow.

mode interaction point is associated with the intersection of the bifurcation lines $\mathbf{A}_{\text {II }}$ and $\mathbf{R}$ of the $1 \alpha$-motion.

\section{The dissipative case}

\subsection{Introduction}

The investigation of the Hamiltonian case has provided us with a sound basis for the next step (towards an explanation of experimental observations of mode competition): the introduction of dissipation. This has already been anticipated in Eq. (1.1), with the $\gamma$-terms representing viscous damping in the pivots; as in Ref. [2], we shall take $\gamma=0.1 \mathrm{~s}^{-1}$. The effects of the dissipation in the linearly coupled case have been described in detail in Ref. [2]. One of these effects is the appearance of quasiperiodic behaviour in the form of attracting limit cycles (born at the Hopf bifurcation lines), 
paving the way for various routes to chaos. For example, the limit cycles are often observed to undergo period-doubling bifurcations leading to chaotic attractors. Another important effect of dissipation is the destruction of the system's symmetry of time reversal $\mathbf{t}$. This puts the $\alpha$ and $\beta$ variations of the various motions into the same symmetry class, enabling them to annihilate each other at the so-called dissipation thresholds. Furthermore, it causes certain Hamiltonian bifurcation lines (namely $\mathbf{B}_{\mathrm{II}}$ and $\mathbf{J}$ ) to unfold into saddle-node lines. These effects are, of course, also present in the case of nonlinear coupling.

It is useful to recall that dissipation also affects the eigenvalues. Complex-conjugate pairs no longer travel on the unit circle, but instead on the so-called reduced circle with radius $e^{-n_{\gamma_{2} / 2}}$, where $n$ denotes the period of the orbit. Thus, in period-doubling bifurcations only one eigenvalue goes through -1 , and in symmetry-breaking (and saddle-node) bifurcations only one eigenvalue goes through $+1 .{ }^{4}$ Hopf bifurcations are still characterized by two eigenvalues of a quadruplet breaking through the unit circle. The labeling of the bifurcation lines in the stability diagrams presented in this section will be identical to that of the previous section; dotted lines, however, will from this point on represent the bifurcation lines of the motions in the nonlinearly coupled Hamiltonian system, i.e., for $L=0.2 \mathrm{~s}^{-2}$ and $\gamma=0$.

The remainder of this section is built up in analogy with the previous one. In Section 3.2 we present the stability diagrams of the 2-motions and the mixed motion of type D. In Section 3.3 we deal with the mixed motions of type A and MP, concentrating on the (neighborhood of) the primary and secondary mode interaction points. Finally, in Section 3.4, we perform a numerical experiment (as in Ref. [2]) to see how the motion types manifest themselves in a parctical situation.

\subsection{The 2-motions and the mixed motion of type $D$}

In Section 2 we saw that, due to the third-order coupling term $L$, both $2 \alpha$ and $2 \beta$ exhibit hard as well as soft versions which annihilate each other along the saddle-node lines $\mathbf{X} \alpha$ and $\mathbf{X} \beta$, respectively. These saddle-node lines can best be regarded as frequency threshold lines along which the solution sheet is folded backwards (cf. Fig. 3), distinguishing an upper 'hard' sheet and a lower 'soft' sheet. The dissipation threshold, which appears with nonzero $\gamma$, is folded along with the sheet; for the soft 2-motions it thus rises with decreasing $\Omega$ and for the hard versions it rises with increasing $\Omega$.

In Fig. 12 we present the stability diagrams for the $2 x$-motion, for $K=1 \mathrm{~s}^{-2}$, $L=0.2 \mathrm{~s}^{-2}$ and $\gamma=0.1 \mathrm{~s}^{-1}$. Note that the figure incorporates data of both the soft

\footnotetext{
${ }^{4}$ This has the convenient consequence that the analysis of the two pitchfork bifurcations occurring simultaneously at the primary mode interaction point (and the associated mode interaction between the $1 \alpha$ and $2 \beta$ motions) can be reduced to a two-dimensional problem by using a technique known as center manifold reduction. This analysis is performed in Appendix B.
} 


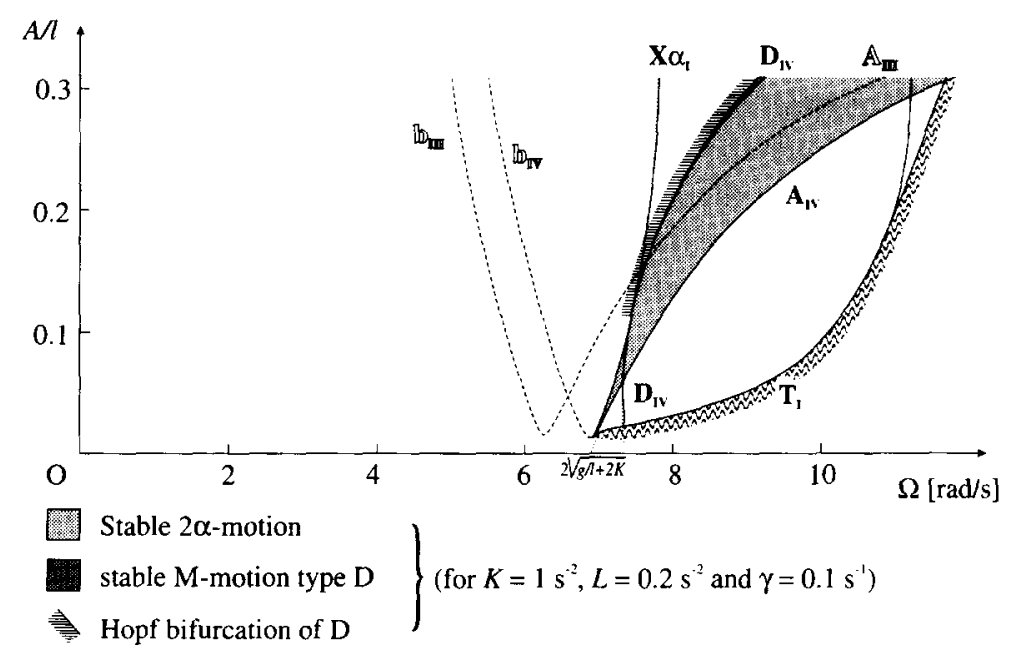

Fig. 12. Stability diagram of the $2 \alpha$-motion and the mixed motion of type D, for $L=0.2 \mathrm{~s}^{-2}$ and $\gamma=0.1 \mathrm{~s}^{-1}$. Note that data of both the soft and the hard $2 \alpha$ has been depicted. The dashed lines represent bifurcation lines of the 0-motion.

and the hard version of $2 \alpha$; in addition, we have included the mixed motion of type D. The soft $2 \alpha$-motion, which for the present values of $L$ and $\gamma$ is the only one that has a stable region, exists between the lines $\mathbf{A}_{\mathrm{JV}}$ and $\mathbf{X} \alpha_{1}$; the hard version exists between lines $\mathbf{T}_{1}$ and $\mathbf{X} \alpha_{I}$ and is at best semi stable, namely in the region enclosed by the lower segments of $\mathbf{X} \alpha_{I}$ and $\mathbf{D}_{I V}$. The frequency threshold $\mathbf{X} \alpha_{I}$ marks the end of the soft and hard versions of $2 \alpha$, and at the dissipation threshold $\mathbf{T}_{\mathrm{I}}$ the $2 \alpha$ - and $2 \beta$-motions annihilate each other. The latter has apparently been folded completely to the right, and the annihilation here concerns only the hard versions of $2 \alpha$ and $2 \beta$. (As noted in the previous subsection the annihilation of $2 \alpha$ and $2 \beta$ is made possible by the absence of time-reversal symmetry, which puts both motions in the same symmetry class, with symmetries RT, ET and RE and 1). Fig. 13 depicts the stability diagram of the $2 \beta$-motion, of which only a hard version exists at the present values of $L$ and $\gamma$ (hence, the absence of a $\beta$ frequency threshold line in the figure).

Qualitatively, the only real change in these stability diagrams brought on by dissipation is the appearance of $\mathbf{T}_{1}$, but this has important quantitative consequences. In the Hamiltonian case $(\gamma=0)$ we saw a whole series of stable regions of the hard $2 \alpha$ and $2 \beta$-motions, and we commented upon their (doubtful) significance from a practical point of view. It turns out that the inclusion of dissipation resolves the matter quite tidily: the 'hard' dissipation threshold is seen to rise rapidly with increasing $\Omega$, and banishes the recurrent stable bands to unrealistically high values of the driving amplitude $A$.

To conclude this subsection, we briefly turn our attention to the most important bifurcation product of the $2 \alpha$-motion, namely the mixed motion type $D$. In the presence of dissipation type D has only the ET symmetry (and 1). In Fig. 12 we see 


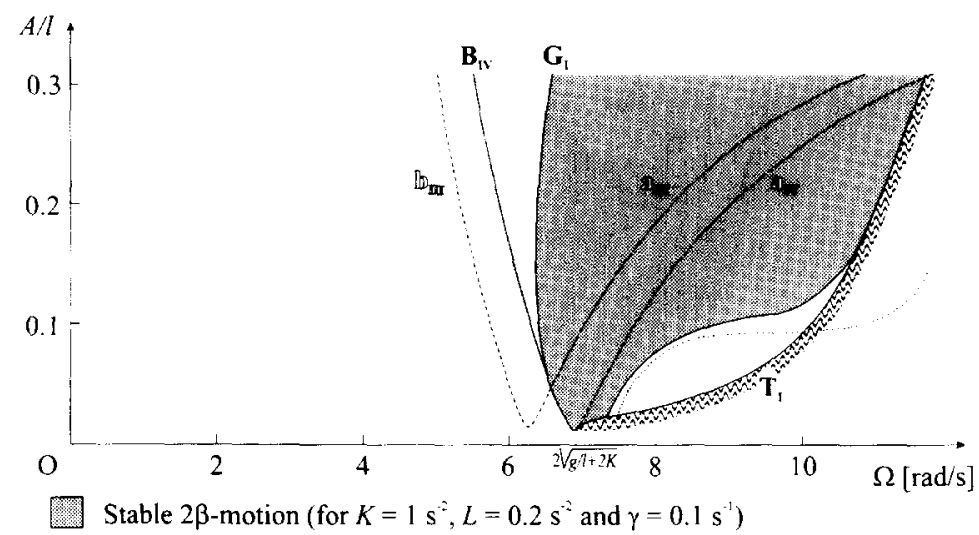

Fig. 13. Stability diagram of the $2 \beta$-motion, for $L=0.2 \mathrm{~s}^{-2}$ and $\gamma=0.1 \mathrm{~s}^{-1}$. For this value of $L$ only a hard version of $2 \beta$ appears. The dashed lines represent bifurcation curves of the 0 -motion.

that type $D$ is stable only in a narrow strip to the left of (the upper part of) its birthline $\mathbf{D}_{\mathrm{IV}}$, but soon loses its stability by means of a Hopf bifurcation.

\subsection{The mixed motions of type $A, B$ and $M P$}

The loss of time-reversal symmetry has put the mixed motions A, MP and ML in the same symmetry class, characterized by only one nontrivial symmetry: RT. One could argue that it is therefore no longer appropriate to designate them with different names. This is correct as far as the ML motion is concerned, which formerly connected MP and A. Indeed, one of the two ML-branches has now become identical to A (in the sense that there exists no bifurcation to distinguish the two) and the other ML-branch has become identical to MP, so we drop the name 'Mixed Link'. The motions A and MP are still to be distinguished, however, in much the same way as one distinguishes between $\alpha$ and $\beta$ versions of a motion: they are separated at least by a saddle-node bifurcation. In Fig. 14 we have drawn the stability diagram of the A-motion and its bifurcation product B, and in Fig. 15 that of the MP-motion. These figures are closely connected, as becomes clear when we take a look at the bifurcational structures (along the arrows marked I and II in Fig. 15), presented in Fig. 16a and Fig. 16b.

To begin with, note that the secondary mode interaction point has been unfolded by the dissipation. The points $\xi_{\mathrm{L}}$ and $\xi_{\mathrm{R}}$ now distinguish the parts of $\mathbf{A}_{\mathrm{V}}$ and $\mathbf{R}_{\mathrm{I}}$ where the $1 \alpha$-motion gains stability in either a super-or a subcritical bifurcation (see also Ref. [2]). Thus, at the line denoted as $\mathbf{R}_{\mathrm{I}, \text { left }}$ we see MP being born semi stable, while at line $\mathbf{R}_{\mathbf{I} \text {.right }}$ it is born stable. Analogously, at $\mathbf{A}_{\mathbf{V} \text {.high }}$ the A-motion is born semi stable, while at $\mathbf{A}_{\mathbf{V} \text {,low }}$ it is born stable.

The semi stable A-branch emanating from $\mathbf{A}_{\mathbf{V} \text {.high }}$ (towards the left) can be followed to lead to the remnant of line $\mathbf{J}$ which, for reasons of compatibility with Ref. [2], has 


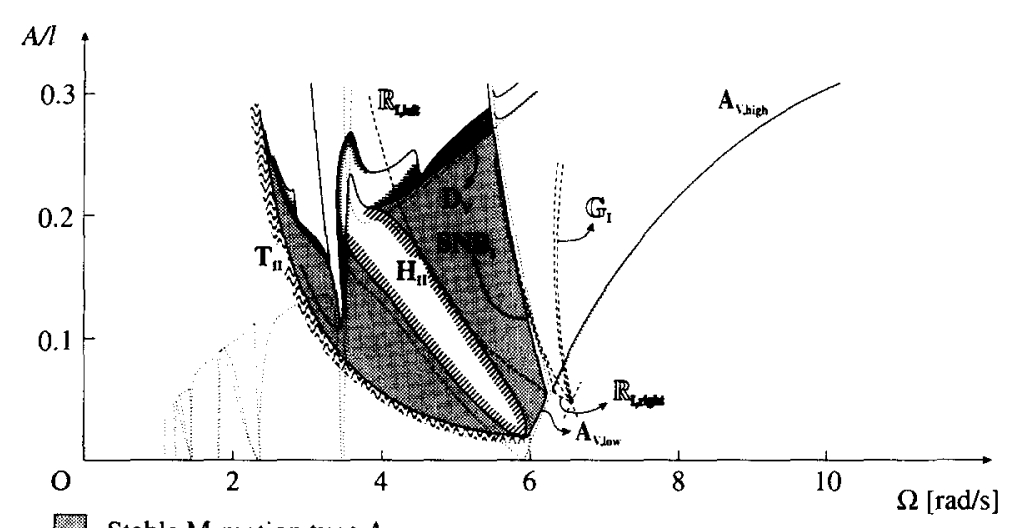

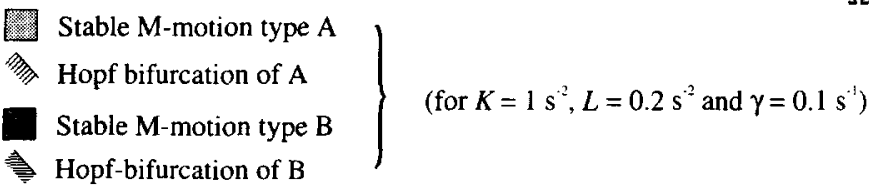

Fig. 14. Stability diagram of the mixed motions of type A and B, for $L=0.2 \mathrm{~s}^{-2}$ and $\gamma=0.1 \mathrm{~s}^{-1}$. The dashed lines represent bifurcation lines of the MP-motion.

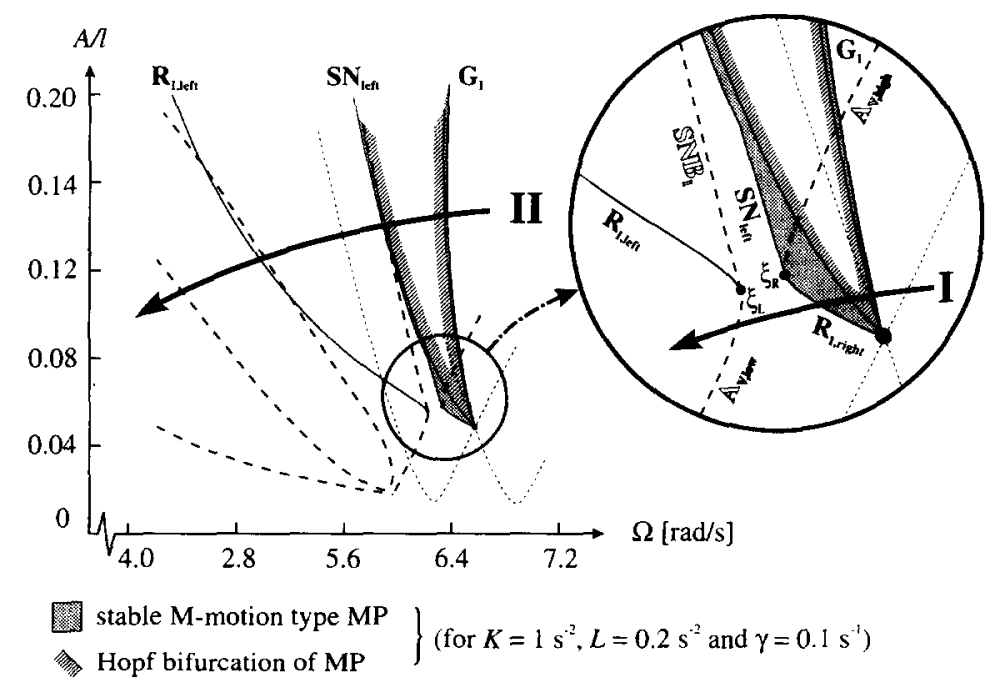

Fig. 15. Stability diagram of the mixed motion of type $M P$, for $L=0.2 \mathrm{~s}^{-2}$ and $\gamma=0.1 \mathrm{~s}^{-1}$. The dashed lines represent bifurcation lines of the A-motion and the 0 -motion.

been denoted as $\mathbf{S} \mathbf{N}_{\text {left }}$. Here a saddle-node bifurcation takes place. The other, stable branch contributing to the bifurcation at $\mathbf{S N}_{\text {left }}$ can be identified as MP and heads to the right towards the $2 \beta$-motion at the line $\mathbf{G}_{\mathbf{I}}$; by the time it reaches line $\mathbf{G}_{\mathbf{I}}$ it has undergone two successive Hopf bifurcations, turning the branch from stable to (fully) unstable to stable again. Likewise, the semi stable MP-branch emanating (upwards) 


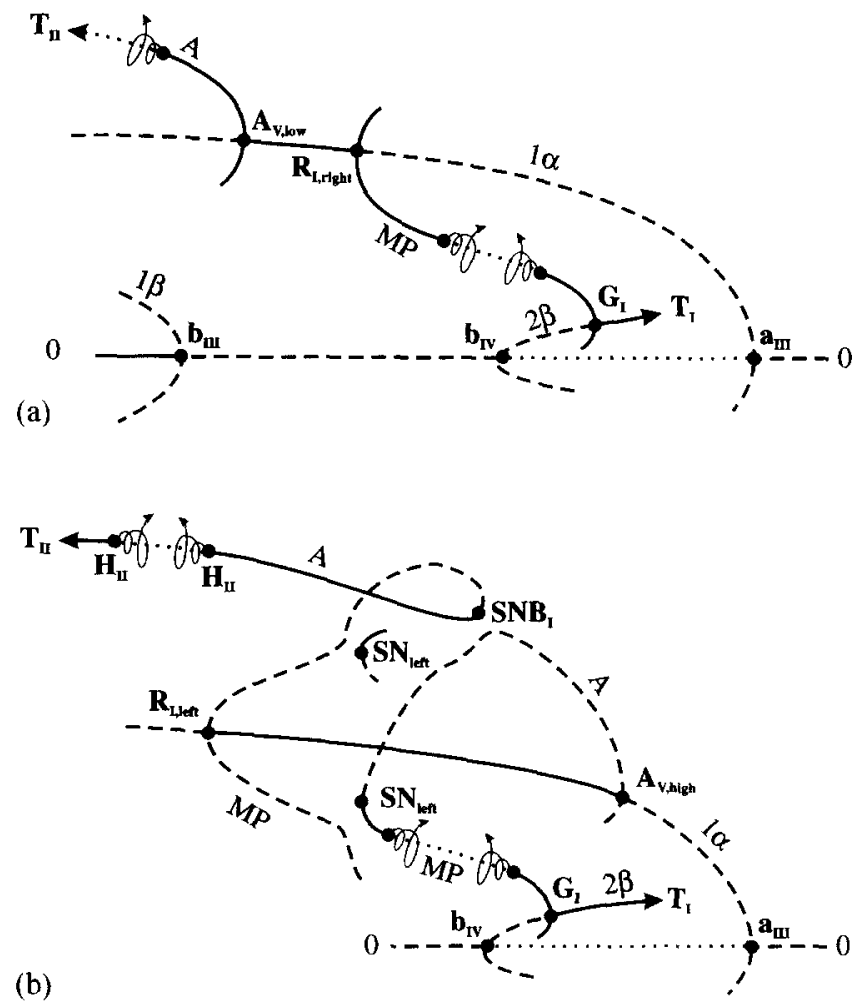

Fig. 16. (a) and (b) Schematic representation of the bifurcational structure along the arrows marked $I$ and II in Fig. 15.

from $\mathbf{R}_{\mathbf{I}, \text { left }}$ can be followed to meet an A-branch in the saddle-node bifurcation along line $\mathbf{S N B}_{1}$, the remnant of line $\mathbf{B}_{\mathrm{II}}$. The (stable) A-branch heads towards the left to finally reach the dissipation threshold $\mathbf{T}_{\mathrm{II}}$, where it annihilates with its $\beta$-counterpart (an A-like motion bifurcated from the $1 \beta$-motion). Before that, however, it has lost and regained stability by means of two Hopf bifurcations.

Along line $\mathbf{A}_{\mathbf{V} \text {,low }}$ we witness the birth (from $1 \alpha$ ) of a stable A-motion. After two Hopf bifurcations (in which it loses and regains stability) this A-motion annihilates with its $\beta$-counterpart at the dissipation threshold $\mathbf{T}_{\text {II }}$. Likewise, along line $\mathbf{R}_{\text {I.right }}$ a stable MP-motion is born from $1 \alpha$, and after two Hopf bifurcations this MP-motion recombines with the $2 \beta$-motion along line $\mathbf{G}_{\mathrm{l}}$, the latter emerging from this 'reverse' symmetry breaking (i.e., symmetry restoring) as a stable motion.

The names of the saddle-node lines $\mathbf{S N}_{\text {left }}$ and $\mathbf{S N B}$ I suggest the existence of an $\mathbf{S N}_{\text {right }}$ and perhaps and $\mathbf{S N B}_{11}$ too. These lines indeed exist for linearly coupled pendulums [2], where line $\mathbf{J}$ unfolds (just as in the present case) into the single saddle-node line $\mathbf{S N}_{\text {left }}$, but $\mathbf{B}_{\mathrm{II}}$ unfold into three saddle-node lines $\mathbf{S N B}_{1}, \mathbf{S N B}_{\mathrm{II}}$ and $\mathbf{S N}_{\text {right }}$, as depicted in Fig. 17. Now, imagine the three saddle-node lines (Fig. 17) to 


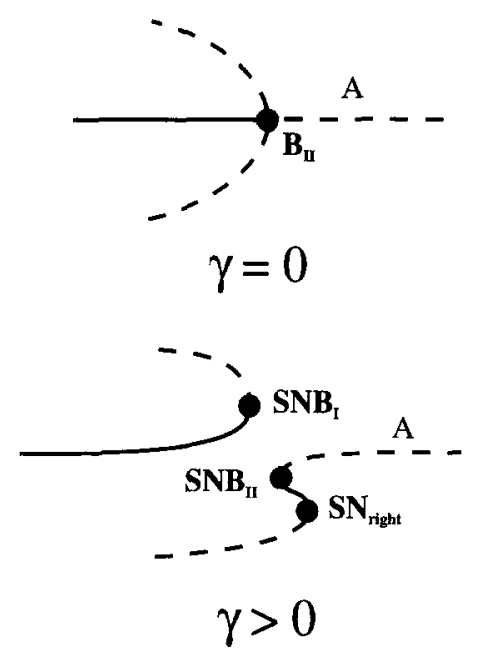

Fig. 17. Schematic representation of the unfolding of the Hamiltonian bifurcation line $\mathbf{B}_{\mathrm{II}}$ into $\mathbf{S N B} \mathbf{B}_{\mathrm{I}}, \mathbf{S N B}_{\mathrm{II}}$ and $\mathbf{S} \mathbf{N}_{\text {right }}$.

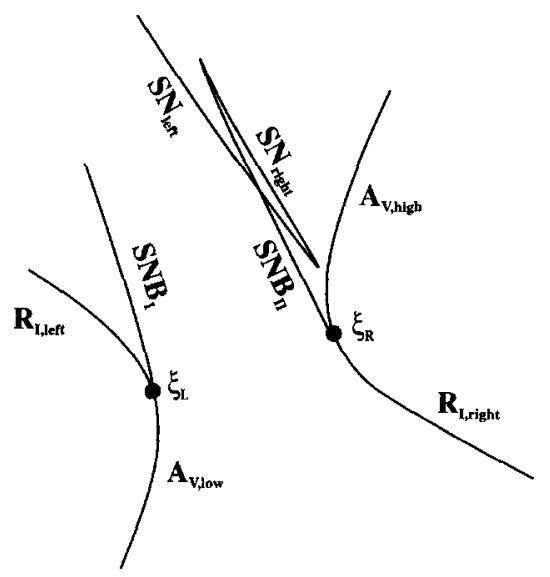

Fig. 18. The possible joining of lines $\mathbf{S N B}$ II, $\mathbf{S N}_{\text {right }}$ and $\mathbf{S N}_{\text {lef } 1}$.

take the place of the single saddle-node line $\mathbf{S N B}_{1}$ in Fig. 16b. Line $\mathbf{S N}_{\text {right }}$ must then lie to the right of both $\mathbf{S N}_{\text {left }}$ and $\mathbf{S N B}$ II. Furthermore, lines $\mathbf{S N B}$ II and $\mathbf{S N}_{\text {right }}$ can cancel each other in this situation, and so can $\mathbf{S N}_{\text {right }}$ and $\mathbf{S} \mathbf{N}_{\text {left }}$. This makes a joining of the lines as sketched in Fig. 18 possible. In this figure, which represents the situation for a small nonlinear coupling term $L$, line $\mathbf{S} \mathbf{N}_{\text {right }}$ exists only in a small part of the $(A, \Omega)$-plane; for some critical value of $L$ the line will disappear from the picture, thereby directly connecting $\mathbf{S N}_{\text {left }}$ and $\mathbf{S N B} \mathbf{B}_{\mathrm{II}}$. This explains the peculiar kink in line $\mathbf{S N}_{\text {left }}$ (now recognized as actually being a blend of $\mathbf{S N B} \mathbf{B}_{\text {II }}$ and $\mathbf{S N}_{\text {left }}$ ) which can be discerned in Fig. 15. 
There are still a few words to say about the stability diagram of the A- and B-motions (Fig. 14). In the first place, we see that the primary and secondary 'bubble' of stability, which in the Hamiltonian case were separated by a gap, have now melted together. At the edges of the former gap the bifurcation of A to B switches from superto subcritical and back again; from the switching points emerge saddle-node lines of the B-motions. (These lines have been indicated in the figure, but without a name). This means that the stable B-regions capping the two bubbles of stability are still disconnected.

There is a connection now, though, between the B-motions per bubble. In the Hamiltonian case there were two types of B-motion bifurcating from the primary bubble: one with RTt symmetry (to the left side of the bubble) and one with $\mathbf{t}$ symmetry (above the bubble). But now, in the presence of dissipation, all B-motions fall into the same symmetry class (with the identity $\mathbf{1}$ as the only remaining element) and this enables them to merge.

In retrospect, the resemblance in the bifurcational lives of the A- and MP-motion is striking. Indeed, the loss of time-reversal symmetry due to the dissipation has established an intimate relationship between the two. Yet there is one important difference, which cannot be left unmentioned: MP forms a direct link between the two 'interacting' normal motions $1 \alpha$ and $2 \beta$ (in the neighborhood of the mode interaction point) whereas A does not. Or, in other words, MP is the common daughter of the two interacting motions whereas $\mathrm{A}$ is the daughter only of $1 \alpha$.

The question whether the direct link (MP) can be stable over the entire interval between $1 x$ and $2 \beta$ is, of course, of great practical interest. For the chosen set of parameters $K=1 \mathrm{~s}^{-2}, L=0.2 \mathrm{~s}^{-2}$ and $\gamma=0.1 \mathrm{~s}^{-1}$ the answer seems to be negative: both endpoints are stable (that is, MP is born stable from $1 \alpha$ as well as from $2 \beta$ ) but the middle part is rendered unstable by two Hopf bifurcations; numerical evidence leads us to believe that the Hopf bifurcation lines (see Fig. 15) stretch out all the way to the primary mode interaction point. If we choose another set of parameter values, however, or if we include additional nonlinearities to the system (e.g. fifth-order coupling terms) we might get a completely stable link; see also Appendix B.

\subsection{A numerical experiment}

Now that we have discussed many of the motions that can occur in the system, it is interesting to study which of them do occur in a practical situation. Just as in Ref. [2] we will therefore perform a numerical experiment, i.e., we release the pendulums from (close to) their downward equilibrium position and examine in which type of motion they eventually settle down. For driving parameters outside the resonance tongues this will obviously be the 0 -motion, but inside the tongues just about anything can happen.

In Fig. 19 we have given the results of this experiment. Some 80000 points in the $(A, \Omega)$-plane are involved, and for each of these points the pendulums have been followed for 20000 driving periods (which in a real-life experiment would take several hours). 


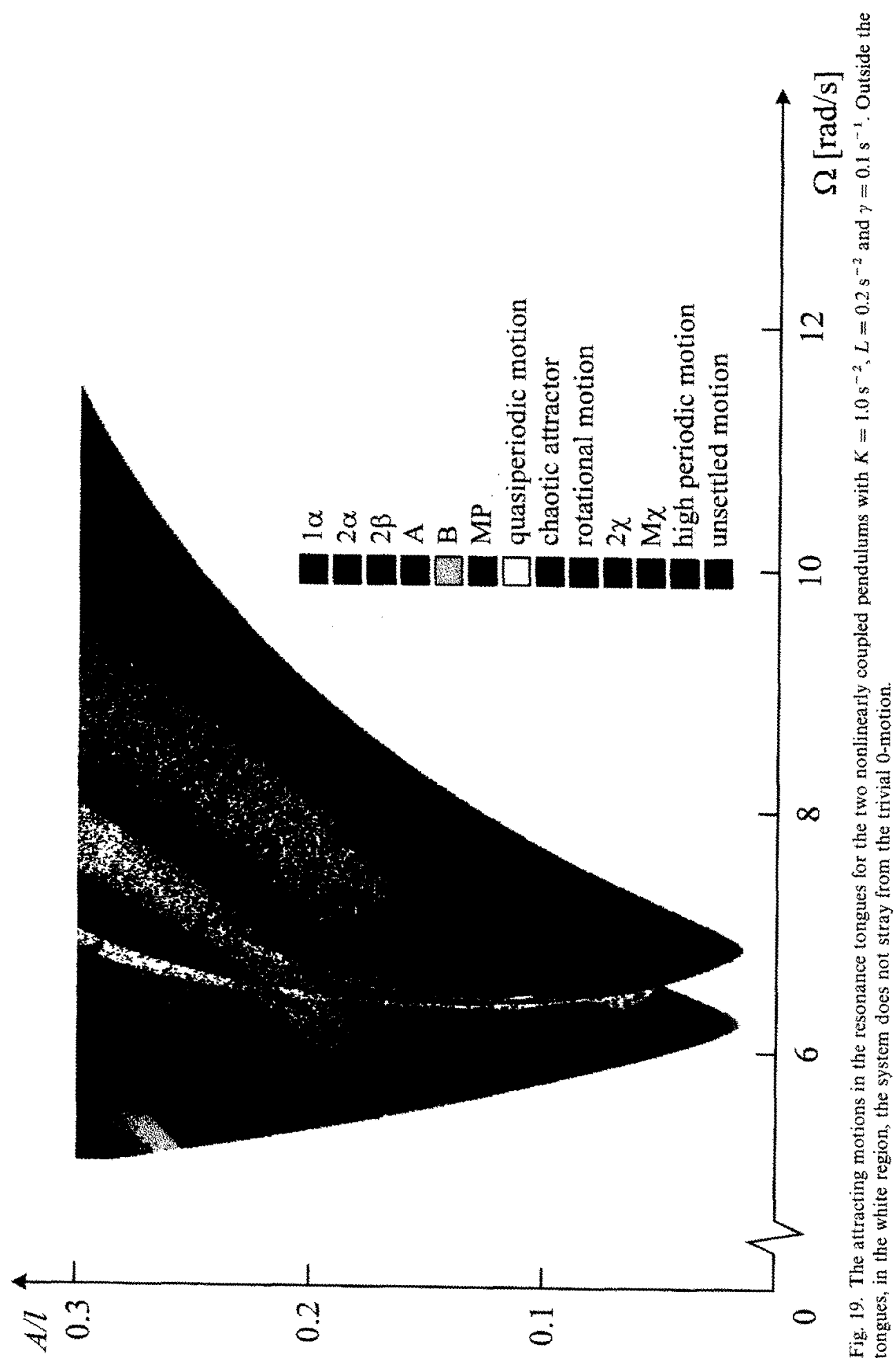


It is gratifying to see that the situation around the primary mode interaction point is precisely as expected. That is, the position of lines $\mathbf{R}_{\mathrm{I} \text {, left }}-\mathbf{A}_{\mathbf{V}, \text { low }}, \mathbf{R}_{\mathbf{I}, \mathrm{right}}-\mathbf{A}_{\mathbf{V} \text {,high }}$ and $\mathbf{G}_{\mathbf{l}}$ (see also Fig. 15) can clearly be recognized in the figure; these lines are the borders of the stable regions of A (orange), $1 \alpha$ (light blue), MP (red) and $2 \beta$ (light green). Also the left Hopf bifurcation line of MP can be recognized, marking the transition from periodic to quasiperiodic behaviour (yellow). In this region we also find perioddoubled limit cycles, frequency-locked orbits and chaotic attractors; the latter correspond to the black dots in Fig. 19. In Fig. 20 we present the transition from a stable MP-motion to a chaotic attractor (and back to a stable MP-motion again) for $A / l=0.057$, that is, for a horizontal path in the $(A, \Omega)$-plane through the lowest black dot. Fig. 20a gives the normal coordinates $\phi_{1}\left(=\vartheta_{1}+\vartheta_{2}\right)$ and $\phi_{2}\left(=\vartheta_{1}-\vartheta_{2}\right)$ of the MP-motion as we follow it from $1 \alpha$ to $2 \beta$, i.e., from the $\phi_{1}$-axis to the $\phi_{2}$-axis. Where the solid line becomes dotted the MP-motion loses its stability and gives way to a limit cycle. Along the dotted part of the line we have marked six points, representing the successive stages in the route to chaos depicted in Fig. 20b-Fig. 20g. At the point marked b, i.e., at $A / l=0.057$ and $\Omega=6.470 \mathrm{rad} / \mathrm{s}$, the experiment yields a limit cycle; at $\mathrm{c}$ this limit cycle has undergone a period-doubling bifurcation; at $\mathrm{d}$ we have still the same period-doubled cycle, but now grown in size; at $\mathrm{e}$ (corresponding to be black dot in the $(A, \Omega)$-plane) the attractor has become chaotic, and it has also recombined with its sister in a symmetry-restoring event; a magnification of the central part of this chaotic attractor is presented as well. So, at $A / l=0.057$ and $\Omega=6.510 \mathrm{rad} / \mathrm{s}$ the pendulums, released from close to the downward equilibrium, end up in a chaotic motion. At $\mathrm{f}$ the chaos is over and the attractor is again a regular limit cycle; at $\mathrm{g}$ the cycle has become considerably smaller and is on the verge of extinction, since MP is about the stabilize again before it connects to the $2 \beta$ motion at the $\phi_{2}$-axis (these last few stages take place within an extremely narrow range of $\Omega$-values). It may be noted that the 'restored' symmetry present in the chaotic attractor has been broken again in the limit cycle of Fig. 20g; this is a necessary prelude to the MP-motion, which does not have this symmetry.

Further away from the primary mode interaction point, close to the left border of the tongues (and halfway up in the diagram), we recognize the saddle-node line $\mathbf{S N B}_{1}$. To the left of this line type A (orange) gradually takes over from $1 \alpha$ (light blue). The transition from A to $\mathbf{B}$ (silver gray) at line $\mathbf{D}_{\mathbf{v}}$ is also clearly visible. In addition to motions of period $2 T$ we also find motions of higher period (dark blue) in this region, which include $1: 3$ resonances of type $A$ (orbits with period $6 T$ ), period-doubled versions thereof (period 12T) and period-doubled B motions (period 4T).

At the other side of the diagram line $\mathbf{D}_{\mathrm{IV}}-\mathbf{X} \alpha_{1}$ can be identified. At $\mathbf{D}_{\mathrm{IV}} 2 \alpha$ (dark green) transfers its stability to the mixed motion of type $\mathrm{D}$; the latter hardly ever appears in this experiment (in fact, only 2 out of the $80000(A, \Omega)$-combinations were found to yield type $D$ ). In its place other motions appear, such as rotations and the so-called $2 \chi$-motions discussed below. It is interesting to note that, although $2 \beta$ 's stable region totally engulfs that of $2 \alpha$, the latter emerges as the dominant motion whenever it is stable. This is due to the fact that the fixed points of $2 \alpha$ lie much closer 


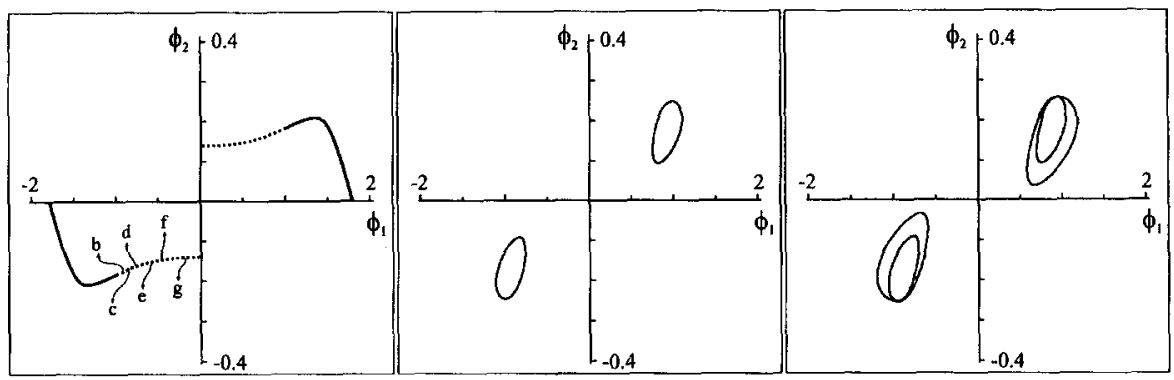
a) $A / l=0.057$
b) $\Omega=6.470 \mathrm{rad} / \mathrm{s}$
c) $\Omega=6.480 \mathrm{rad} / \mathrm{s}$

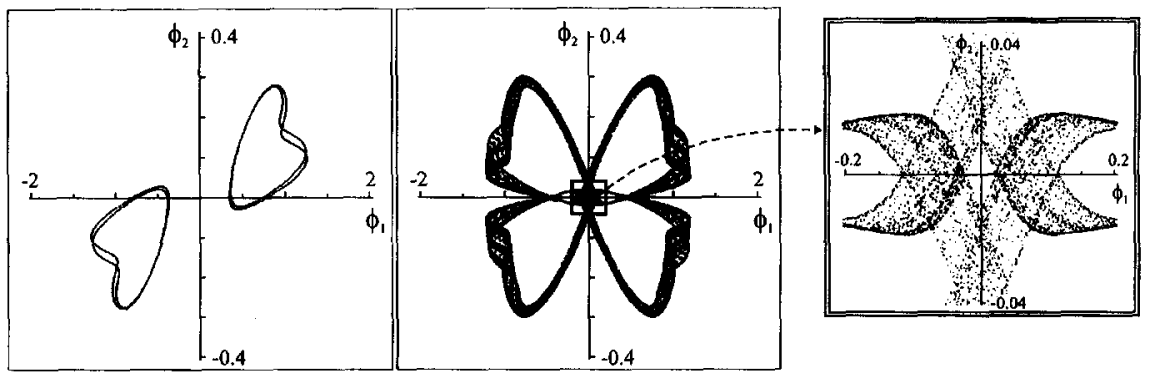
d) $\Omega=6.490 \mathrm{rad} / \mathrm{s}$
e) $\Omega=6.510 \mathrm{rad} / \mathrm{s}$

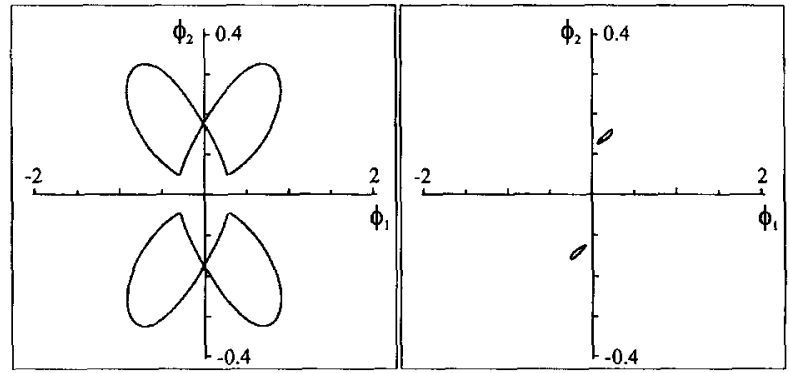

f) $\Omega=6.520 \mathrm{rad} / \mathrm{s} \quad$ g) $\Omega=6.536 \mathrm{rad} / \mathrm{s}$

Fig. 20. The transition from the $1 \alpha$-motion to the $2 \beta$-motion along a horizontal path, at $A / l=0.057$, through the $(A, \Omega)$-plane. (a) depicts the changing position of the MP-motion, expressed in terms of the normal coordinates $\phi_{1}$ and $\phi_{2}$; the motion is stable along the solid part of the line and undergoes a Hopf bifurcation where the line becomes dotted. The next stages of the transition, depicted in (b)- $(\mathrm{g})$ do not concern the MP-motion itself but the development of the limit cycle born at the Hopf bifurcation. See the text for a more detailed description.

to the origin of stroboscopic phase space than those of $2 \beta$, and the basin of attraction of $2 \alpha$ will therefore dominate the neighborhood of the origin.

In the middle part of the diagram we see scattered occurrences of periodic MPmotions (red), bounded by line $\mathbf{G}_{\mathrm{I}}$ and the neighboring Hopf bifurcation line, as well as quasiperiodic (yellow) and frequency-locked (dark blue) versions of MP. (One should realize that the Hopf-bifurcated products of MP do not necessarily lie within 


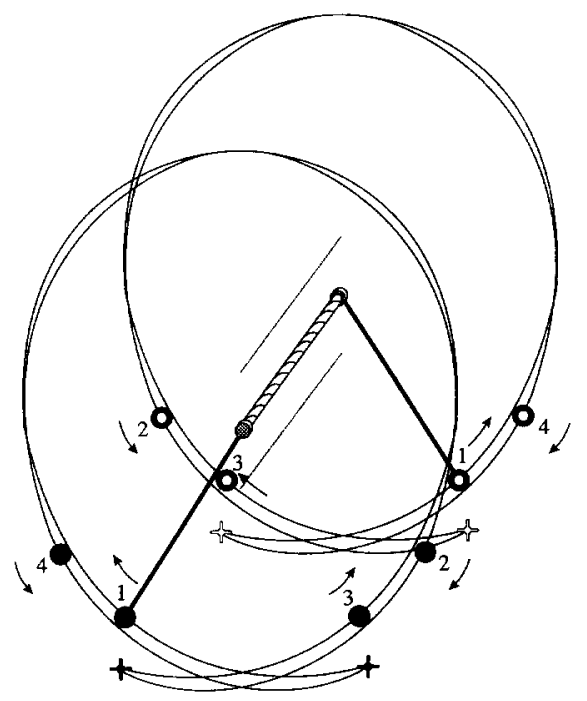

(a)

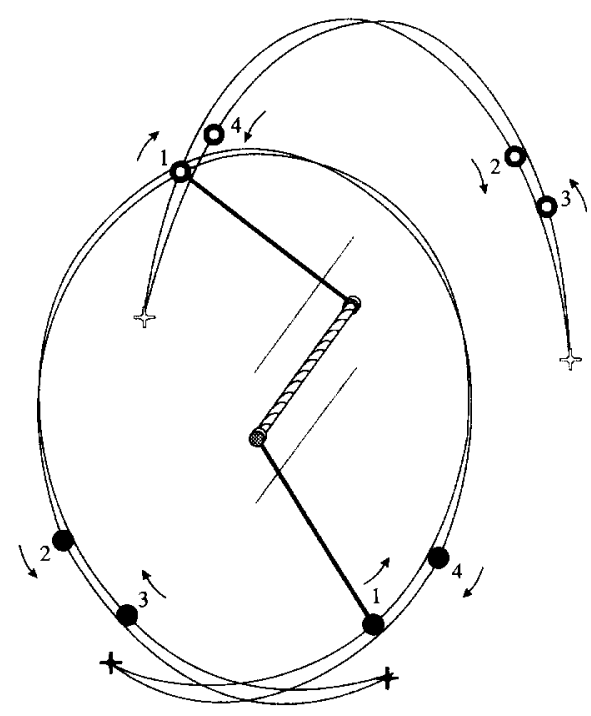

(b)

Fig. 21. (a) Stable $2 T$-periodic $2 \chi$ motion, for $\Omega=8.81 \mathrm{rad} / \mathrm{s}$ and $A / l=0.27$. (b) Stable $2 T$-periodic $\mathbf{M} \gamma$ motion, for $\Omega=5.37 \mathrm{rad} / \mathrm{s}$ and $A / l=0.30$. The numbers 1 and 3 denote the position of the pendulums the moment the bar of suspension passes its middle position in the upwards direction; numbers 2 and 4 give the positions when the bar of suspension passes its middle position going downward. The crosses indicate the moments of stroboscopic sampling (when the suspension bar goes through its lowest position).

the region bounded by MP's two Hopf bifurcation lines.) All in all, the MP-motion beautifully serves as intermediate motion between $2 \beta$ (light green) and $1 \alpha$, when the former gradually turns over its dominance to the latter.

Apart from the motion types discussed so far, Fig. 19 also contains other motions. For example, rotational motions (light purple) play a significant role in this experiment; indeed, they turn up not only for large values of the driving amplitude (as in the case of linear coupling [2]) but also for values of $A$ comparable to those of the mode interaction region. A second example is the motion designated as $2 \chi$ (dark purple), depicted in Fig. 21a. The period of this motion is $2 T$ and the pendulums move in exact counterphase, with $\vartheta_{1}+\vartheta_{2}=2 \pi$; in other words, it is a 2-motion around the upward equilibrium. It is found just to the left of the $2 \alpha$-motion's region, in competition with the rotational motions (light purple). Both types of motion come into play in the region where $2 \alpha$ turns over its dominance to $2 \beta$; i.e. the system hesitates between $2 \alpha$ and $2 \beta$ and the pendulums find an attractive alternative. Traveling towards the left in the diagram the $2 \chi$-motion bifurcates, giving rise to a family of mixed motions collectively denoted as $\mathbf{M} \chi$ (dark gray); one example of such a motion is shown in Fig. 21b. We see that the dark gray bands of stable $\mathbf{M} \chi$ motions are interlaced with yellow bands of quasiperiodic behavior, associated (predominantly) with limit cycles 
that have bifurcated from $\mathbf{M} \chi$. Indeed, these higher regions of the diagram are full of motions which we have not seen before. This shows once more the richness of the system, but since these motions do not relate directly to the topic of this paper (mode interaction) we shall not pursue them any further.

It is interesting to compare Fig. 19 with the corresponding figure for linear coupling i.e., with Fig. 25 in Ref. [2]. The profound effect of the nonlinear coupling term then becomes apparent. For one thing, in the linear case only one type of 2-motion was ever stable (the $2 \alpha$-motion) whereas now both $2 \alpha$ and $2 \beta$ occupy a considerable part of the right-hand resonance tongue. As a side effect there is also a transition region between the two, in which other motions rear their heads (even at comparatively low values of the driving amplitude). Also, in the higher parts of the diagram the nonlinear coupling term is seen to bring about a lot of changes. Our main interest, however, lies with the effect of the third-order coupling on the motions near the primary mode interaction point. For linear coupling the MP-motion hardly appeared in the experiment (only a very narrow strip of stable MP, and no quasiperiodic orbits associated with it) and $2 \alpha$ was seen to give up its dominance to $1 \alpha$ directly, i.e., without any intermediate motion. For nonlinear coupling, on the other hand, MP manifests itself strongly and we observe an abundance of limit cycles (and even chaos) derived from it. So we see that very simple picture around the primary mode interaction point for linear coupling (when one almost exclusively finds the highly symmetrical $1 \alpha$ - and $2 \alpha$ motions) has changed into a rather complex one with motions that are much less symmetric, including quasiperiodic and chaotic ones.

\section{Summary and conclusions}

This paper is the third in a series on the dynamics of two coupled, parametrically driven pendulums. In earlier work we examined in detail the prominent motions and possible routes to chaos in the case of linear coupling between the pendulums, both for the Hamiltonian [1] and the dissipative system [2]. In this paper we studied the effects of a nonlinear (third-order) term in the coupling, with coefficient $L$, both for the Hamiltonian and the dissipative case. The main reason to add a nonlinear term to the coupling was to make the mode interaction less degenerate. In the linearly coupled case we did find mode interaction between the normal motions $1 \alpha$ and $2 \beta$, but the region of this interaction in the $(A, \Omega)$-plane was extremely small due to a degeneracy. That is to say, lines $\mathbf{R}$ and $\mathbf{G}$ ran very close to each other for $L=0$. The degeneracy is lifted, and lines $\mathbf{R}$ and $\mathbf{G}$ are separated, by the inclusion of a third-order coupling term; this is explained in Appendix B.

Some motions, namely the 0 -motion and the 1 -motions, are not affected by the nonlinearity in the coupling. (This also means that line $\mathbf{R}$, where the MP-motion bifurcates from the $1 \alpha$-motion, is left unchanged). This is a direct consequence of the fact that the spring is not stretched at all when the system performs these motions. Other motions are only mildly affected; these include the mixed motions of type A, 
$\mathrm{B}$ and $\mathrm{D}$. They remain qualitatively the same and their regions of stability are shifted only slightly. But the rest of the motions, in particular, the 2-motions and the mixed motion of type MP, is severely affected.

Let us first review the effects on the 2-motions. We find that these motions do not exist anymore for values of the driving frequency $\Omega$ below certain frequency thresholds, which in the $(A, \Omega)$-plane appear as saddle-node lines and are called $\mathbf{X} \alpha$ and $\mathbf{X} \beta$. At $\mathbf{X} \alpha$ we witness the birth of a hard and a soft variation of the $2 \alpha$-motion, and at $\mathbf{X} \beta$ the same happens for the $2 \beta$-motion. The soft motions have the (familiar) property that their amplitude decreases with increasing frequency. The hard variations exhibit a peculiar recurrent pattern of stable regions stretching from $\mathbf{X} \alpha$ (and $\mathbf{X} \beta$, respectively) all the way to infinite $\Omega$, but these regions are of little practical importance since they are pushed to extremely high values of $A$ when dissipation is added.

On increasing the third-order coupling coefficient $L$ the lines $\mathbf{X} \alpha$ and $\mathbf{X} \beta$ are pushed further and further to the right in the $(A, \Omega)$-plane; for $L=0.2 \mathrm{~s}^{-2}$ the line $\mathbf{X} \beta$ has been pushed even beyond $\mathbf{B}_{1}$, the birthline of the $2 \beta$-motion. This means that, near the primary mode interaction point, the $2 \beta$-motion now becomes fully stable at line $\mathbf{G}$, a situation not encountered in the case of linear coupling. Line $\mathbf{G}$ has been swept to the right by the action of the third-order term in the coupling.

This has also important consequences for the MP-motion, the main agent of the mode interaction in our system, since this motion is born from $2 \beta$ at line $\mathbf{G}$. Specifically, MP's region of existence (between lines $\mathbf{R}$ and $\mathbf{G}$ ) is widened considerably; this is in fact the central point of the present paper. The position of line $\mathbf{G}$ can be controlled by adjusting the coefficient $L$ while line $\mathbf{R}$, where MP is born from $1 \alpha$, is not affected by $L$. The mode interaction would even be more important if the MP-motion would be stable over its entire region of existence, but this is not the case in our system. MP is born stable at both ends (at line $\mathbf{R}$ and at line $\mathbf{G}$ ) but in the intermediate region it is rendered unstable by means of Hopf bifurcations.

We have also found a second instance of mode interaction, not between two normal motions but between the mixed motions MP and A, via a motion called ML. This secondary mode interaction is however much less important than the (primary) mode interaction between $1 \alpha$ and $2 \beta$, since it only occurs in the absence of dissipation.

The general rule is that mode interaction cannot take place between just any two motions, but only between those which have a common daughter in the symmetry hierarchy of the system. More specifically, two motions can (directly) interact only if the symmetry groups associated with them have a common subgroup. It is then easily seen that $1 \alpha$ and $2 \beta$ can interact (via MP) both in the Hamiltonian and in the dissipative system, whereas MP and A can only interact (via ML) in the Hamiltonian system. Indeed, in the Hamiltonian case the symmetry groups of $1 \alpha$ $\left(Z_{2}(\mathrm{RT}) \times Z_{2}(\mathrm{E}) \times Z_{2}(\mathrm{t})\right)$ and $2 \beta\left(Z_{2}(\mathrm{RT}) \times Z_{2}(\mathrm{ET}) \times Z_{2}(\mathrm{Tt})\right)$ have the symmetry group of $\mathrm{MP}\left(\boldsymbol{Z}_{2}(\mathbf{R T}) \times \boldsymbol{Z}_{2}(\mathbf{E t})\right)$ as their common subgroup; the groups of MP and $\mathrm{A}\left(\boldsymbol{Z}_{2}(\mathbf{R T}) \times \boldsymbol{Z}_{2}(\mathbf{t})\right)$ in turn have the symmetry group of $\mathrm{ML}\left(\boldsymbol{Z}_{2}(\mathbf{R T})\right)$ as their common subgroup. Introduction of dissipation removes the time-reversal symmetry from the equations of motion and this causes the elements containing $t$ to disappear from the 
various symmetry groups. The groups of $1 \alpha$ and $2 \beta$ then still contain a common subgroup, $\boldsymbol{Z}_{2}(\mathbf{R T})$, and interaction between the two via MP can (and does) still take place. The mode interaction between $A$ and MP via ML no longer occurs, however, since all three motions now have the same symmetry group $Z_{2}(\mathbf{R T})$. We will come back in detail to these symmetry considerations in a forthcoming article [10]; in the present paper we have laid the foundation for this by listing the individual symmetry elements of the various motions.

The present paper has provided yet another insight, which has to do with the fact that the nonlinear terms in our equations of motion can be divided into two categories: the nonlinearity of the oscillators themselves and the nonlinearity in the coupling. This neat division of the nonlinear terms proves to be very helpful in unraveling the intricacies of the mode interaction. The nonlinearity in the coupling, as we have seen, controls the width of the mode interaction region (i.e., the position of line $\mathbf{G}$ with respect to $\mathbf{R}$ ). The nonlinear terms in the oscillators, on the other hand, control the position of the interaction region with respect to the mode interaction point. For our oscillators the first nonlinear term (the cubic term in $\sin \vartheta=\vartheta-\frac{1}{6} \vartheta^{3}+\cdots$ ) has the opposite sign of the linear term; in other words, the pendulum is a soft oscillator. This means that the $1 \alpha$-motion is born towards the left in the $(A, \Omega)$-plane and hence that line $\mathbf{R}$ emanates towards the left from the primary mode interaction point. If we would have used hard oscillators instead, for which the first nonlinear term has the same sign as the linear one, line $\mathbf{R}$ would have emanated towards the right.

In conclusion, we have made clear why it is necessary to have a nonlinearity in the coupling to get an appreciable region of mode interaction in our system. In this context it may be noted that in the experiments of Ciliberto and Gollub $[3,4]$ and Skeldon et al. [8,9], producing some of the finest illustrations of mode interaction to date, the coupling between the modes is also (highly) nonlinear.

\section{Acknowledgements}

We would like to thank S. Oosthoek for helping us with the installation of Linux on our computers, thereby doubling our computational capacity.

\section{Appendix A. On the nature of the mixed motions}

In this appendix we will show how knowledge of the one-pendulum system can provide us with a good idea of what the various mixed motions bifurcating from the normal motions look like. We will concentrate on those which in the main text have been dubbed type A, MP and type D.

Let us begin by writing down the equation of motion for a single, parametrically driven pendulum (in the absence of dissipation):

$$
\ddot{\vartheta}+f(t) \sin \vartheta=0 \text {, }
$$


with $f(t)=\left(g+A \Omega^{2} \cos \Omega t\right) / l$, as in the main text. The prominent motions in this system are the 0 -motion (which, for convenience, is regarded as a motion of period 2 with respect to the driving) and its direct bifurcation products; their stability is governed by the eigenvalues of the linearized twice-iterated stroboscopic map, denoted as $P$. The Hamiltonian nature of Eq. (A.1) ensures that the product of the two eigenvalues is equal to 1 . From the highly symmetrical nature of the 0 -motion and its direct bifurcation products it follows that, for these motions, the matrix $P$ has the general form (as can easily be checked by working out the explicit expression of $P$, as in Appendix A of Ref. [1]):

$$
P=\left[\begin{array}{ll}
X & V \\
W & X
\end{array}\right], \quad \text { with } P=X^{2}-V W=1 .
$$

The symmetry also inhibits the motions discussed here (including the ' $2 T$-periodic' 0 -motion) to undergo a period-doubling bifurcation [10], and we need only consider symmetry-breaking bifurcations, where the two eigenvalues are at +1 . In terms of $P$ this corresponds to one of the following two forms:

$$
\text { (a): } P=\left[\begin{array}{ll}
1 & V \\
0 & 1
\end{array}\right] \text { or }(b): P=\left[\begin{array}{ll}
1 & 0 \\
W & 1
\end{array}\right] \text {. }
$$

Having established the general form of $P$ at bifurcation we further note that the eigenvectors of $P$ can be used to indicate the position of the newly born motions. Now, the eigenvectors of the matrices defined by Eq. (A.3) are of a very simple form. In Fig. A.1 we have drawn the bifurcation lines of the 0 -motion (lines $A$ and $B$ ) and of the symmetrical $\alpha$-motion bifurcating from it (line $\mathrm{D}$ ). Along these lines we show the form of $P$ and its eigenvectors, which indicate the direction of the bifurcation in the (two-dimensional) stroboscopic phase space. It is immediately clear from the figure

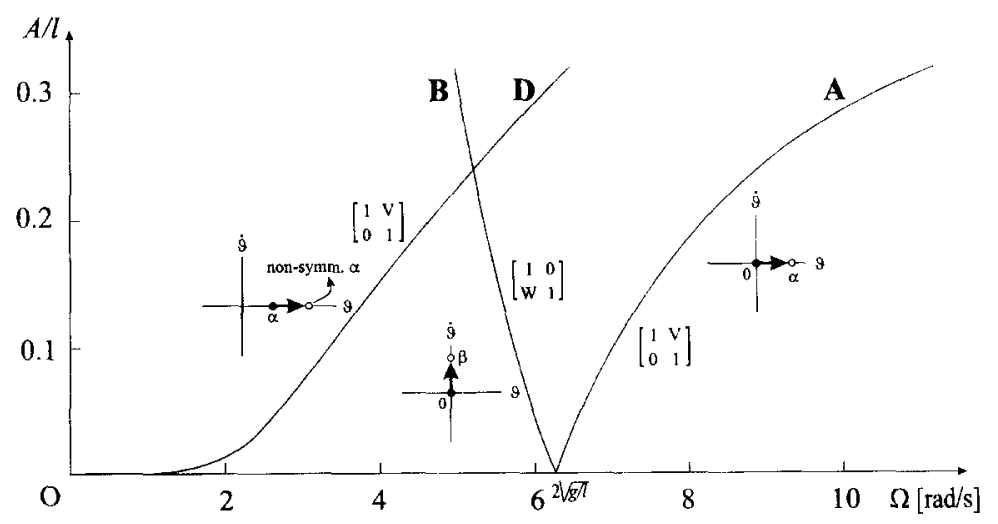

Bifurcation lines of the 0-motion and the $\alpha$-motion

Fig. A.1. Bifurcation curves of the 0 -motion and of the symmetrical $x$-motion. 
that we get an $\alpha$-motion at line $\mathbf{A}$, a $\beta$-motion at line $\mathbf{B}$, and a nonsymmetrical $\alpha$-motion at line $\mathbf{D}$.

Let us now generalize this to four dimensions. The normal motions (including the 0 -motion) can be regarded as essentially one-pendulum motions, and hence it is convenient to introduce normal coordinates defined as

$$
\begin{aligned}
& \phi_{1} \equiv \vartheta_{1}+\vartheta_{2}, \\
& \phi_{2} \equiv \vartheta_{1}-\vartheta_{2} .
\end{aligned}
$$

The equations of motion (viz. Eq. (1.1) in the main text) then become

$$
\begin{aligned}
& \ddot{\phi}_{1}+2 f(t) \sin \frac{1}{2} \phi_{1} \cos \frac{1}{2} \phi_{2}=0, \\
& \ddot{\phi}_{2}+2 f(t) \sin \frac{1}{2} \phi_{2} \cos \frac{1}{2} \phi_{1}+2 K \phi_{2}+2 L \phi_{2}^{3}=0 .
\end{aligned}
$$

In these coordinates the linearized, twice-iterated stroboscopic map will, for the motions discussed, have the general form (see also Ref. [1]):

$$
P=\left[\begin{array}{cccc}
X_{1} & V_{1} & 0 & 0 \\
W_{1} & X_{1} & 0 & 0 \\
0 & 0 & X_{2} & V_{2} \\
0 & 0 & W_{2} & X_{2}
\end{array}\right]
$$

The upper left block governs, as indicated by the indices, the eigenvalues in the 1-direction, while the lower right block provides the 2-eigenvalues.

When the $1 \alpha$-motion, with fixed points $\left(\phi_{1}, \dot{\phi}_{1}, \phi_{2}, \dot{\phi}_{2}\right)=(\mu, 0,0,0)$, gives birth to mixed motions, its 2-eigenvalues will be at +1 , and we find for $P$ :

$$
\text { (a): } P=\left[\begin{array}{cccc}
X_{1} & V_{1} & 0 & 0 \\
W_{1} & X_{1} & 0 & 0 \\
0 & 0 & 1 & V_{2} \\
0 & 0 & 0 & 1
\end{array}\right] \text { or (b): } P=\left[\begin{array}{cccc}
X_{1} & V_{1} & 0 & 0 \\
W_{1} & X_{1} & 0 & 0 \\
0 & 0 & 1 & 0 \\
0 & 0 & W_{2} & 1
\end{array}\right] \text {, }
$$

with eigenvectors (in the 2-direction) given by

$$
\text { (a): }\left(\begin{array}{c}
\phi_{1} \\
\dot{\phi}_{1} \\
\phi_{2} \\
\dot{\phi}_{2}
\end{array}\right)=\left(\begin{array}{c}
0 \\
0 \\
1 \\
0
\end{array}\right) \quad \text { or (b): }\left(\begin{array}{c}
\phi_{1} \\
\dot{\phi}_{1} \\
\phi_{2} \\
\dot{\phi}_{2}
\end{array}\right)=\left(\begin{array}{l}
0 \\
0 \\
0 \\
1
\end{array}\right) \text {. }
$$

So there are two possibilities. In the first case (a) the new fixed point will have a $\phi_{1}$ and a $\phi_{2}$-component: $(\mu, 0, v, 0)$. The corresponding mixed motion can thus be viewed as a $1 \alpha$-motion with an (initially small) component in the $\phi_{2}$-direction: the pendulums move in phase but with different amplitudes. The motion is known to us as type A. In the second case (b) the new fixed point has coordinates $(\mu, 0,0, v)$, and the corresponding mixed motion can be viewed as a $1 \alpha$-motion with an (initially small) component in 


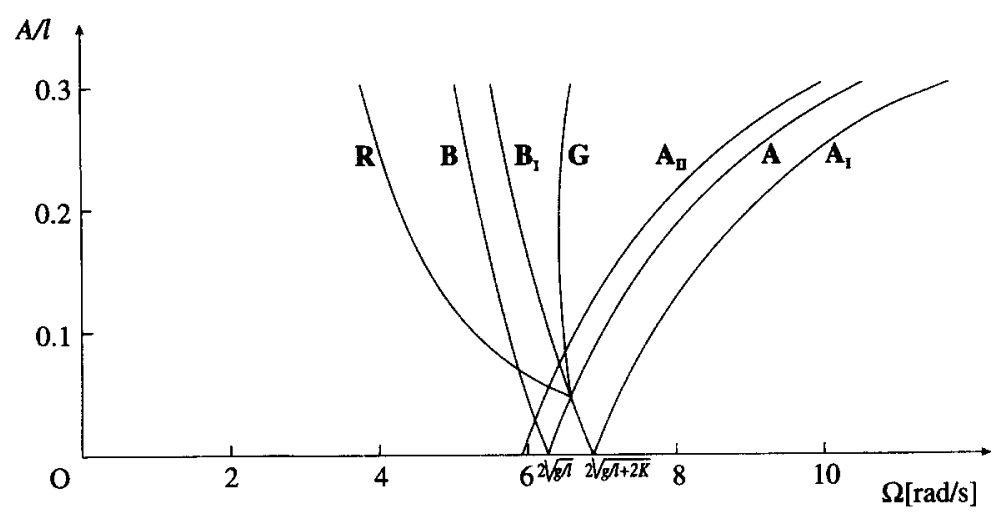

Various bifurcation lines of the 0 -motion, the $1 \alpha$-motion and the $2 \beta$-motion, for $K=1 \mathrm{~s}^{-2}, L=0.2 \mathrm{~s}^{-2}$.

Fig. A.2. Bifurcation lines of the $0-, 1 \alpha-, 2 \alpha$ - and $2 \beta$-motion, for $L=0.2 \mathrm{~s}^{-2}$.

the $\dot{\phi}_{2}$-direction. This means that the phase difference between the pendulums will not be constant; this is the familiar MP-motion.

Now, along which lines do the above two possibilities occur? The answer can be found by inspection of Fig. A.2, where we have depicted the bifurcation lines of the 0-, $1 \alpha$-, $2 \alpha$ - and $2 \beta$-motion. As indicated by the labels, line $\mathbf{A}_{\mathrm{II}}$ is related (via a continuous deformation) to line $\mathbf{A}$ of Fig. A.1, which was characterized by the form of $P$ given in Eq. (A.3a). Remembering that we are dealing with the 2 -eigenvalues of the $1 \alpha$-motion (i.e., with the lower right block in the four-dimensional version of $P$ ) it follows that line $\mathbf{A}_{\mathrm{II}}$ is characterized by Eqs. (A.7a) and (A.8a). The mixed motion born here is therefore type A.

From Fig. A.2 we can also deduce that the second possibility, characterized by Eqs. (A.7b) and (A.8b), occurs at line R. After all, line $\mathbf{R}$ is connected to the intersection point of lines $\mathbf{A}$ and $\mathbf{B}_{\mathrm{l}}$, where the lower right block of the four-dimensional $P$ has the form as given in Eq. (A.3b). (This can be inferred from the fact that line $\mathbf{B}_{\mathbf{I}}$ is a continuous deformation of line $\mathbf{B})$. The mixed motion born from $1 \alpha$ along line $\mathbf{R}$ is therefore MP.

The MP-motion can also be born as a daughter of $2 \beta$, along line $\mathbf{G}$. The $2 \beta$-motion is characterized by a fixed point on the $\dot{\phi}_{2}$-axis, i.e. $\left(\phi_{1}, \dot{\phi}_{1}, \phi_{2}, \dot{\phi}_{2}\right)=(0,0,0, v)$. Furthermore, just like line $\mathbf{R}$, line $\mathbf{G}$ connects to the intersection point of $\mathbf{A}$ and $\mathbf{B}_{\mathbf{l}}$ and hence the upper left block of the four-dimensional $P$, providing the 1-eigenvalues, has the form given in Eq. (A.3a). We thus get, once more, a fixed point with coordinates $(\mu, 0,0, v)$; the corresponding MP-motion can be regarded as a $2 \beta$-motion with an (initially small) component in the $\phi_{1}$-direction.

Let us finally turn to the mixed motion type $D$, which is born from the $2 \alpha$-motion, i.e., from $(0,0, v, 0)$. This happens at line $D_{I}$, and it can easily be verified that the upper 
left block of the four-dimensional $P$ (providing the 1-eigenvalues) has the form given by Eq. (A.3a). Type D thus has the fixed-point form $(\mu, 0, v, 0)$ and can be viewed as a $2 \alpha$-motion with an (initially small) $\phi_{1}$-component, i.e., as a counter-phase motion of the two pendulums centered around some nonzero angle.

In conclusion, we have given an easy recipe for getting an impression of what the mixed motions bifurcating from the normal motions look like. One might be tempted to think that knowledge of the general form of one fixed point is enough to establish which type of mixed motion we are dealing with. This is however not correct. For instance, type $\mathrm{A}$ and type $\mathrm{D}$ both correspond to the form $(\mu, 0, v, 0)$. We need extra information to classify them; one may get this by retracing the orbit to its birthline (as above) or one may take into consideration the orbits' second fixed point too (thereby using the symmetry properties to distinguish between the various mixed motions).

\section{Appendix B. Analysis of the primary mode interaction point}

\section{B.1. Introduction}

In this appendix we derive the bifurcational structure around the primary mode interaction point in the presence of dissipation. Thanks to the fact that this point is defined by two (simultaneous) bifurcations of the trivial solution, the 0 -motion, the derivation can be carried out analytically. We will proceed in three steps. First, we remove the explicit time dependence from the equations of motion by using a method known as averaging. Subsequently, we eliminate two of the four dimensions of phase space from the problem by restricting the dynamics to the so-called center manifold; in the presence of dissipation two dimensions are sufficient to capture all the essential features of the system's behavior. In the third step we present the normal form of the averaged restricted equations to finally establish the bifurcational structure near the primary mode interaction point. Throughout this appendix bold roman characters denote vectors or matrices, non-bold lowercase roman and Greek characters represent scalars, and uppercase (bold) Greek characters stand for manifolds in $\mathbf{R}^{4}$.

\section{B.2. Step one: averaging the equations of motion}

First of all, since we set out to study the nature of the interaction of the two normal modes $1 \alpha$ and $2 \beta$, it is useful to switch to normal coordinates, defined by

$$
\begin{aligned}
& \phi_{1} \equiv \vartheta_{1}+\vartheta_{2}, \\
& \phi_{2} \equiv \vartheta_{1}-\vartheta_{2} .
\end{aligned}
$$


Written as a set of four first-order differential equations, the equations of motion (viz. Eq. (1.1) in the main text) then become

$$
\dot{\Phi} \equiv \frac{d}{d t}\left(\begin{array}{c}
\phi_{1} \\
\dot{\phi}_{1} \\
\phi_{2} \\
\dot{\phi}_{2}
\end{array}\right)=\left(\begin{array}{c}
\dot{\phi}_{1} \\
-2 f(t) \sin \frac{1}{2} \phi_{1} \cos \frac{1}{2} \phi_{2}-\gamma \dot{\phi}_{1} \\
\dot{\phi}_{2} \\
-2 f(t) \sin \frac{1}{2} \phi_{2} \cos \frac{1}{2} \phi_{1}-2 K \phi_{2}-2 L \phi_{2}^{3}-\gamma \dot{\phi}_{2}
\end{array}\right) .
$$

In these coordinates a 1 -motion is characterized by $\phi_{2}(t)=0$ for all $t$, while the 2-motions have the property $\phi_{1}(t)=0$.

We can get rid of the explicit time-dependence in our problem by making use of the technique of averaging [13]. The periodic solutions of a system of the form

$$
\dot{\mathbf{x}}=\varepsilon \mathbf{f}(\mathbf{x}, t)+O\left(\varepsilon^{2}\right),
$$

with $\varepsilon$ some (small) parameter, correspond with fixed points of the so-called averaged equation:

$$
\dot{\overline{\mathbf{x}}}=\varepsilon \overline{\mathbf{f}}(\overline{\mathbf{x}}), \quad \text { with } \overline{\mathbf{f}}(\mathbf{x})=\frac{1}{T} \int_{0}^{T} \mathbf{f}(\mathbf{x}, t) d t
$$

The system defined by Eq. (B.2) is not yet in the right form for averaging. We therefore invoke, with a certain amount of foresight, the following transformations:

$$
A \rightarrow \varepsilon A, \quad \gamma \rightarrow \varepsilon \gamma \quad \text { and } \Phi \rightarrow \sqrt{\varepsilon \Phi}
$$

and restrict ourselves to the case of small $\varepsilon$, so that we can dismiss terms of $O\left(\varepsilon^{2}\right)$. With these substitutions the system of Eq. (B.2) becomes:

$$
\dot{\Phi}=\left(\begin{array}{c}
\dot{\phi}_{1} \\
-\omega_{1}^{2} \phi_{1}+\varepsilon\left(\frac{1}{8} \omega_{1}^{2} \phi_{1} \phi_{2}^{2}+\frac{1}{24} \omega_{1}^{2} \phi_{1}^{3}-A \Omega \cos (\Omega t) \phi_{1}-\gamma \dot{\phi}_{1}\right) \\
\dot{\phi}_{2} \\
-\omega_{2}^{2} \phi_{2}+\varepsilon\left(\frac{1}{8} \omega_{1}^{2} \phi_{2} \phi_{1}^{2}+\frac{1}{24} \omega_{1}^{2} \phi_{2}^{3}-A \Omega \cos (\Omega t) \phi_{2}-2 L \phi_{2}^{3}-\gamma \dot{\phi}_{2}\right)
\end{array}\right),
$$

where we have used $\omega_{1}=\sqrt{g / l}$ and $\omega_{2}=\sqrt{g / l+2 K}$. These equations describe two near-harmonic, slightly damped oscillators with eigenfrequencies $\omega_{1}$ and $\omega_{2}$, coupled only by nonlinear terms. Since we are investigating motions is parametric resonance we expect to find (near-sinsusoidal) solutions with frequency $\frac{1}{2} \Omega$, i.e., with half the driving frequency. This, in turn, suggests that we might try the following coordinate 
transformation (a generalization of the so-called van der Pol transformation [14]):

$$
\mathbf{x} \equiv\left(\begin{array}{c}
x_{1} \\
x_{2} \\
x_{3} \\
x_{4}
\end{array}\right)=\frac{-1}{\Omega}\left[\begin{array}{cccc}
-\Omega \cos \frac{1}{2} \Omega t & 2 \sin \frac{1}{2} \Omega t & 0 & 0 \\
\Omega \sin \frac{1}{2} \Omega t & 2 \cos \frac{1}{2} \Omega t & 0 & 0 \\
0 & 0 & -\Omega \cos \frac{1}{2} \Omega t & 2 \sin \frac{1}{2} \Omega t \\
0 & 0 & \Omega \sin \frac{1}{2} \Omega t & 2 \cos \frac{1}{2} \Omega t
\end{array}\right]\left(\begin{array}{c}
\phi_{1} \\
\dot{\phi}_{1} \\
\phi_{2} \\
\dot{\phi}_{2}
\end{array}\right) .
$$

In the new coordinates we find that the system of Eq. (B.6) is of the form of Eq. (B.3) when both $\Omega^{2}-4 \omega_{1}^{2}$ and $\Omega^{2}-4 \omega_{2}^{2}$ are small enough (i.e., they must be $O(\varepsilon)$ ). This means that $K$, the linear part of the coupling, must be small. When these conditions are met we can proceed to integrate over $T=4 \pi / \Omega$, and find for the averaged equation:

$$
\dot{\overline{\mathbf{x}}}=\mathbf{A} \overline{\mathbf{x}}+\mathbf{F}(\overline{\mathbf{x}}),
$$

where the linear part is determined by the matrix $\mathbf{A}$ :

$$
\mathbf{A}=\frac{\Omega}{2}\left[\begin{array}{cccc}
-\frac{\gamma}{\Omega} & A+\frac{1}{2}-\frac{2 \omega_{1}^{2}}{\Omega^{2}} & 0 & 0 \\
A-\frac{1}{2}+\frac{2 \omega_{1}^{2}}{\Omega^{2}} & -\frac{\gamma}{\Omega} & 0 & 0 \\
0 & 0 & -\frac{\gamma}{\Omega} & A+\frac{1}{2}-\frac{2 \omega_{2}^{2}}{\Omega^{2}} \\
0 & 0 & A-\frac{1}{2}+\frac{2 \omega_{2}^{2}}{\Omega^{2}} & -\frac{\gamma}{\Omega}
\end{array}\right]
$$

and the nonlinear part is defined by the function $\mathbf{F}$ :

$$
\mathbf{F}(\mathbf{x})=\frac{\omega_{1}^{2}}{32 \Omega}\left(\begin{array}{c}
\left(x_{1}^{2}+x_{2}^{2}\right) x_{2}+x_{2} x_{3}^{2}+3 x_{2} x_{4}^{2}+2 x_{1} x_{3} x_{4} \\
-\left(x_{1}^{2}+x_{2}^{2}\right) x_{1}-x_{1} x_{4}^{2}-3 x_{1} x_{3}^{2}-2 x_{2} x_{3} x_{4} \\
\left(1-\frac{48 L}{\Omega^{2}}\right)\left(x_{3}^{2}+x_{4}^{2}\right) x_{4}+x_{1}^{2} x_{4}+3 x_{2}^{2} x_{4}+2 x_{1} x_{2} x_{3} \\
-\left(1-\frac{48 L}{\Omega^{2}}\right)\left(x_{3}^{2}+x_{4}^{2}\right) x_{4}-x_{2}^{2} x_{3}-3 x_{1}^{2} x_{3}-2 x_{1} x_{2} x_{4}
\end{array}\right) .
$$

Note that, due to the symmetry of the system, there are no terms of even order in the averaged equations. That is, the equations are odd in $\mathbf{x}$.

At the primary mode interaction point (i.e., at the intersection point of the two resonance tongues) two of the four eigenvalues of matrix $\mathbf{A}$ will be equal to zero. (Remember that we are dealing with the eigenvalues of a continuous time system here, 
and not with a time-discrete mapping as in the main text). This conditions can be used to calculate the values of $A$ and $\Omega$ at the intersection point. One finds

$$
\Omega_{c}=\sqrt{2 \omega_{1}^{2}+2 \omega_{2}^{2}}, \quad A_{c}=\frac{\sqrt{\left(\omega_{1}^{2}-\omega_{2}^{2}\right)^{2}+\Omega^{2} \gamma^{2}}}{\Omega^{2}},
$$

in accordance with previous results given in Appendix B of Ref. [2].

\section{B.3. Step two: projection onto the center manifold}

Having removed the explicit time dependence we are left with four spatial dimensions. It so happens that only two of these are needed to capture the essence of the dynamics. This is due to the fact that, at the primary mode interaction point, there are only two zero eigenvalues (the critical eigenvalues) while the rest is nonzero; to be specific, the other two have negative real part. These negative eigenvalues correspond to trivial attraction in phase space onto the so-called center manifold $A$, where all of the essential dynamics takes place. At the fixed point (the origin of phase space) the center manifold is tangent to the plane $\Psi^{\mathrm{c}}$ spanned by the critical eigenvectors $\mathbf{c}_{1}$ and $\mathbf{c}_{2}$.

Now, in order to find the equations of motion on the center manifold we begin by projecting the four-dimensional phase space of Eq. (B.2) onto the critical plane $\Psi^{\mathrm{c}}$ and its complement $\Psi^{\text {s }}$ (the plane spanned by the remaining, stable eigenvectors $\mathbf{s}_{1}$ and $\mathbf{s}_{2}$ ). The projection onto $\Psi^{\mathrm{c}}$ can be written as a linear combination of the critical eigenvectors: $u c_{1}+v c_{2}$. The center manifold can then be characterized by the two scalars $u$ and $v$ and a vector $\mathbf{s}$, which is an element of $\Psi^{\mathrm{s}}$ and (due to the tangency of the center manifold to $\Psi^{\mathrm{c}}$ ) is a second-order polynomial in $u$ and $v$. That is, $\Delta:\{u, v, \mathbf{s}\} \mid \mathbf{s}=\mathbf{s}(u, v)$, with $\mathbf{s} \in \Psi^{\mathrm{s}}$. The situation has been sketched in Fig. B.1.

Any vector $\mathbf{x} \in \mathbf{R}^{4}$ in the four-dimensional phase space can be written as follows:

$$
\mathbf{x}=u \mathbf{c}_{1}+v \mathbf{c}_{2}+\mathbf{s}
$$

where $u \mathbf{c}_{1}+v \mathbf{c}_{2}$ is the part of $\mathbf{x}$ that lies in $\Psi^{\mathrm{c}}$, and $\mathbf{s}$ is the part which belongs to $\Psi^{\prime}$. Now, the Fredholm alternative [15] states that every $\mathbf{s} \in \Psi^{s}$ is perpendicular to the

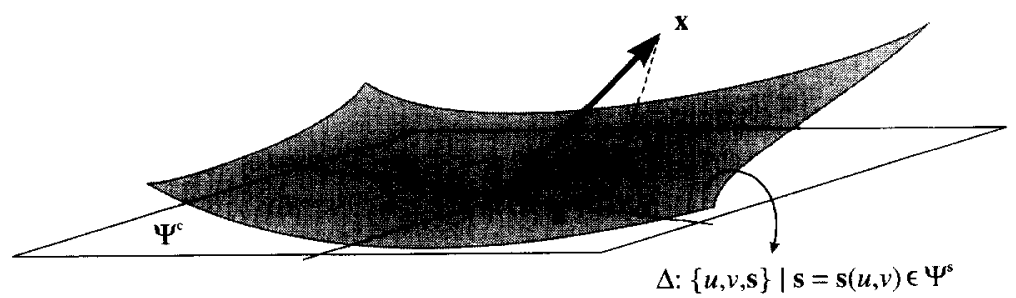

Fig. B.1. Projection of an arbitrary four dimensional vector $\mathbf{x}$ onto the eigenplanes $\Psi^{c}$ and $\Psi^{\star}$, and schematic representation of the center manifold $\Delta$ as a function of $u$ and $u$. Note that vector $\mathbf{s}$ represents the two-dimensional linear eigenspace $\Psi^{\mathrm{s}}$. 
two critical eigenvectors $t_{1}$ and $t_{2}$ of the transposed matrix $A^{T}$, i.e., $t_{1} \cdot s=t_{2} \cdot \mathbf{s}=0$. In fact, an eigenvector of the transposed matrix lies, in our case, perpendicular to those of the original matrix $\mathbf{A}$, the eigenvector corresponding to the same eigenvalue excepted. Thus, one also has $\mathbf{t}_{1} \cdot \mathbf{c}_{2}=\mathbf{t}_{2} \cdot \mathbf{c}_{1}=0$, and (with the proper normalization) $\mathbf{t}_{1} \cdot \mathbf{c}_{1}=\mathbf{t}_{2} \cdot \mathbf{c}_{2}=1$. We may therefore write

$$
\begin{aligned}
& \mathbf{t}_{1} \cdot \mathbf{x}=\mathbf{t}_{1} \cdot\left(u \mathbf{c}_{1}+v \mathbf{c}_{2}+\mathbf{s}\right)=u \mathbf{t}_{1} \cdot \mathbf{c}_{1}+v \mathbf{t}_{1} \cdot \mathbf{c}_{2}+\mathbf{t}_{1} \cdot \mathbf{s}=u, \\
& \mathbf{t}_{2} \cdot \mathbf{x}=\mathbf{t}_{2} \cdot\left(u \mathbf{c}_{1}+v \mathbf{c}_{2}+\mathbf{s}\right)=u \mathbf{t}_{2} \cdot \mathbf{c}_{1}+v \mathbf{t}_{2} \cdot \mathbf{c}_{2}+\mathbf{t}_{2} \cdot \mathbf{s}=v .
\end{aligned}
$$

These are (conveniently simple) expressions for the coefficients $u$ and $v$ which, together with s, may be interpreted as new coordinates:

$$
\begin{aligned}
& u=\mathbf{t}_{1} \cdot \mathbf{x}, \\
& v=\mathbf{t}_{2} \cdot \mathbf{x}, \\
& \mathbf{s}=\mathbf{x}-\left(\mathbf{t}_{1} \cdot \mathbf{x}\right) \mathbf{c}_{1}-\left(\mathbf{t}_{2} \cdot \mathbf{x}\right) \mathbf{c}_{2} .
\end{aligned}
$$

The next step is then to find expressions for the time derivatives of these new coordinates. For $u$ we find, with the use of Eq. (B.8) (dropping the bar-sign for notational convenience):

$$
\dot{u}=\mathbf{t}_{1} \cdot \dot{\mathbf{x}}=\mathbf{t}_{1} \cdot(\mathbf{A x}+\mathbf{F}(\mathbf{x}))=\mathbf{t}_{\mathbf{1}} \cdot \mathbf{A x}+\mathbf{t}_{\mathbf{1}} \cdot \mathbf{F}(\mathbf{x}),
$$

which, realizing that $\mathbf{y} \cdot \mathbf{A x}=\mathbf{A}^{\mathrm{T}} \mathbf{y} \cdot \mathbf{x}$ (for any $\mathbf{x}, \mathbf{y} \in \mathbf{R}^{4}$ ) and that $\mathbf{A}^{\mathrm{T}} \mathbf{t}_{1}=0$, reduces to

$$
\dot{u}=\mathbf{t}_{1} \cdot \mathbf{F}(\mathbf{x})=\mathbf{t}_{1} \cdot \mathbf{F}\left(u \mathbf{c}_{1}+v \mathbf{c}_{2}+\mathbf{s}\right) .
$$

The time derivatives of $v$ and $\mathbf{s}$ can be found analogously, resulting in the following set of equations:

$$
\begin{aligned}
\dot{u}= & \mathbf{t}_{1} \cdot \mathbf{F}\left(u \mathbf{c}_{1}+v \mathbf{c}_{2}+\mathbf{s}\right), \\
\dot{v}= & \mathbf{t}_{2} \cdot \mathbf{F}\left(u \mathbf{c}_{1}+v \mathbf{c}_{2}+\mathbf{s}\right), \\
\dot{\mathbf{s}}= & \mathbf{A s}+\mathbf{F}\left(u \mathbf{c}_{1}+v \mathbf{c}_{2}+\mathbf{s}\right) \\
& -\left(\mathbf{t}_{1} \cdot \mathbf{F}\left(u \mathbf{c}_{1}+v \mathbf{c}_{2}+\mathbf{s}\right)\right) \mathbf{c}_{1} \\
& -\left(\mathbf{t}_{2} \cdot \mathbf{F}\left(u \mathbf{c}_{1}+v \mathbf{c}_{2}+\mathbf{s}\right)\right) \mathbf{c}_{2} .
\end{aligned}
$$

Thus, we have rewritten the averaged equations in the new 'coordinates' $u, v$ and $\mathbf{s}$. We shall now proceed to combine Eq. (B.18) with an expression for $A$ to obtain the desired center manifold dynamics.

Since the vector $\mathbf{s}$ appearing in the expression of the center manifold $A$ is a secondorder function of $(u, v)$, we may write it in a Taylor expansion as

$$
\mathbf{s}=\frac{1}{2} \mathbf{d}_{u^{2}} u^{2}+\frac{1}{2} \mathbf{d}_{v^{2}} v^{2}+\mathbf{d}_{u v} u v+\text { h.o.t., }
$$

where the Taylor coefficients $\mathbf{d}_{u^{2}}, \mathbf{d}_{v^{2}}$ and $\mathbf{d}_{w}$ are (constant) vectors and h.o.t. stands for higher-order terms. Note that $\mathbf{s}$ and $\mathbf{d}_{u^{2}}, \mathbf{d}_{v^{2}}, \mathbf{d}_{u v}$ all belong to the stable eigenplane $\Psi^{\mathrm{s}}$. The center manifold dynamics is now obtained by combining the expression for $\Delta$, 
Eq. (B.19), with the equations of motion in the new coordinates, Eq. (B.18). The general recipe is to substitute Eq.(B.19) (plus Eqs. (B.18a) and (B.18b)) in Eq.(B.18c), and equate terms of same order in $u$ and $v$, to get explicit expressions for $\mathbf{d}_{u^{2}}, \mathbf{d}_{v^{2}}$ and $\mathbf{d}_{u}$ and thereby for $\mathbf{s}$. This expression for $\mathbf{s}$ is then substituted in the argument of $\mathbf{F}$ in Eqs. (B.18a) and (B.18b), to finally obtain the functions $\dot{u}(u, v)$ and $\dot{v}(u, v)$, which describe the center manifold dynamics. Fortunately, however, the symmetries of the system help us to avoid much of the calculations necessary in the general case. In fact. the second-order Taylor coefficients $\mathbf{d}_{u^{2}}, \mathbf{d}_{v^{2}}$ and $\mathbf{d}_{u v}$ all turn out to vanish.

As already mentioned, the function $\mathbf{F}$ given by Eq. (B.10) contains only terms of third order. The leading terms in the Taylor expansion of $\mathbf{F}$ in Eq. (B.18) will therefore be of third order in $u$ and $v$ (with $\mathbf{s}$ also expressed in terms of $u$ and $v$, as in Eq. (B.19)). Indeed, not just any third-order combination of $u$ and $v$ will do: the dependence of $\dot{u}$ on $u$ must be odd, and the same holds for the dependence of $\dot{v}$ on $v$. This is a direct consequence of the fact that the first two lines of $\mathbf{F}$ are odd in $x_{1}$ and $x_{2}$, and the third and fourth line are odd in $x_{3}$ and $x_{4}$. (This is related to the RT symmetry of the $1 \alpha$-and $2 \beta$-motion of the original system). We can therefore immediately write Eq. (B.18) as

$$
\begin{aligned}
& \dot{u}=\mathbf{t}_{1} \cdot\left(\mathbf{F}_{u^{3}}, u^{3}+\mathbf{F}_{u v^{2}} \boldsymbol{u} v^{2}\right)+\text { h.o.t. } \\
& \dot{v}=\mathbf{t}_{2} \cdot\left(\mathbf{F}_{v^{3}}, v^{3}+\mathbf{F}_{u^{2}} u^{2} v\right)+\text { h.o.t., } \\
& \dot{\mathbf{s}}=\mathbf{A s}+\mathbf{F}_{u^{3}} u^{3}+\mathbf{F}_{v^{3}} v^{3}+\mathbf{F}_{\boldsymbol{u}} v^{2} u v^{2}+\mathbf{F}_{u^{2}} u u^{2} v \\
& -\left(\mathbf{t}_{1} \cdot\left(\mathbf{F}_{u^{3}} u^{3}+\mathbf{F}_{v^{3}} v^{3}+\mathbf{F}_{u} !^{2} u v^{2}+\mathbf{F}_{u^{2} v^{2}} u^{2} v\right)\right) \mathbf{c}_{1} \\
& -\left(\mathbf{t}_{2} \cdot\left(\mathbf{F}_{u^{3}} u^{3}+\mathbf{F}_{v^{3}} v^{3}+\mathbf{F}_{u r^{2}} u v^{2}+\mathbf{F}_{u^{2} v^{2}} u^{2} v\right)\right) \mathbf{c}_{2}+\text { h.o.t., }
\end{aligned}
$$

with the Taylor coefficients given by

$$
\begin{aligned}
& \mathbf{F}_{u^{3}}=\left.\frac{1}{6} \frac{\partial^{3} \mathbf{F}\left(u \mathbf{c}_{1}+v \mathbf{c}_{2}+\mathbf{s}(u, v)\right)}{\partial u^{3}}\right|_{u, v=0}, \\
& \mathbf{F}_{u t^{2}}=\left.\frac{1}{2} \frac{\partial^{3} \mathbf{F}\left(u \mathbf{c}_{1}+v \mathbf{c}_{2}+\mathbf{s}(u, v)\right)}{\partial u \partial v^{2}}\right|_{u, v=0}, \\
& \mathbf{F}_{r^{3}}=\left.\frac{1}{6} \frac{\partial^{3} \mathbf{F}\left(u \mathbf{c}_{1}+v \mathbf{c}_{2}+\mathbf{s}(u, v)\right)}{\partial v^{3}}\right|_{u, r=0}, \\
& \mathbf{F}_{u^{2} r}=\left.\frac{1}{2} \frac{\partial^{3} \mathbf{F}\left(u \mathbf{c}_{1}+v \mathbf{c}_{2}+\mathbf{s}(u, v)\right)}{\partial u^{2} \partial v}\right|_{u, v=0} .
\end{aligned}
$$

Since the critical eigenvectors $\mathbf{c}_{1}$ and $\mathbf{c}_{2}$ can be calculated from the expression for $\mathbf{A}$, Eq. (B.9), we are left with only one unknown: we do not (yet) have the precise functional form of $\mathbf{s}=\mathbf{s}(u, v)$ in the argument of $\mathbf{F}$ in Eq. (B.21). It turns out, however, that $\mathbf{s}$ can simply be omitted from Eq. (B.21). To see this, let us first differentiate the Taylor expansion of s, Eq. (B.19), with respect to time

$$
\dot{\mathbf{s}}=\mathbf{d}_{u^{2}} u \dot{u}+\mathbf{d}_{v^{2}} v \dot{v}+\mathbf{d}_{u v}(\dot{u} v+u \dot{v})+\text { h.o.t. }
$$


We already know from Eqs. (B.20a) and (B.20b) that $\dot{u}$ and $\dot{v}$ are of third order in $(u, v)$, and we thus conclude from Eq. (B.22) that $\dot{\mathbf{s}}$ is of fourth order. Putting this information into Eq. (B.20c) it is easily seen that As, and hence s itself is at least of third order in $(u, v) .{ }^{5}$ We, once again, use the fact that $\mathbf{F}$ is of third order in its argument $\mathbf{x}$ to conclude that $\mathbf{s}$ contributes to the equation for $\dot{u}$ and $\dot{v}$ (see Eq. (B.18a, b)) through cross-terms of at least order five in $(u, v)$, which vanish in the third-order derivatives; we can therefore simply omit s from Eq. (B.21). Note that, by these arguments, we can describe the center manifold dynamics (i.e., Eq. (B.20a, b)) without actually having to establish an explicit expression for $\Delta$.

In fact, to low order, the center manifold dynamics can always be derived without an explicit expression for $\Delta$, i.e., without taking into account the vector function $\mathbf{s}$. This can be demonstrated as follows. Suppose that the function $\mathbf{F}$ is of order $\rho$ in $(u, v)$. The tangent nature of the center manifold ensures that the leading terms in $\mathbf{s}$ as given by (Eq. (B.19)) are at least of second order. Since $\dot{u}$ and $\dot{v}$ are at least of order $\rho$ (the same order as F), we know from Eq. (B.22) that $\dot{\mathbf{s}}$ is at least of order $\rho+1$, and that $\mathbf{s}$ is thus at least of order $\rho$. The contribution of $\mathbf{s}$ (via the argument of $\mathbf{F}$ ) to the expressions for $\dot{u}$ and $\dot{v}$ will therefore be at least of order $2 \rho-1$, and vanishes in any derivative of order $2 \rho-2$ or lower. This argument makes determination of the reduced equations to lowest order a fairly simple process. After all, the critical eigenvectors of $\mathbf{A}\left(\mathbf{c}_{i}\right)$ and those of $\mathbf{A}^{\mathrm{T}}\left(\mathbf{t}_{i}\right)$ can be found in a straightforward manner from the expression of the matrix $\mathbf{A}$ (cf. Eq. (B.9)), and the lowest-order Taylor coefficients in the expansions of $\dot{u}$ and $\dot{v}$ then follow by using this knowledge in the expression for $\mathbf{F}$ (cf. Eq. (B.10)).

In order to give a proper analysis of the bifurcational structure around the primary mode interaction point we will find it necessary to include a bifurcation parameter in the reduced equations of motion, and for this purpose we choose variations of the driving amplitude, i.e., $A-A_{c}$ (cf. Eq. (B.11)). The derivation of the reduced equations is the same as above, provided we write $A-A_{c}$ as $x_{5}$, the 'ffifth-phase space coordinate':

$$
\mathbf{x}=\left(x_{1}, x_{2}, x_{3}, x_{4}, A-A_{c}\right)
$$

The inclusion of $x_{5} \equiv A-A_{c}$ will, of course, change the form of $\mathbf{A}$ and $\mathbf{F}$ in Eq. (B.8) somewhat, since $A$ appears explicitly in A. More precisely, every occurrence of $A$ in matrix $\mathbf{A}$ will be replaced by $A_{c}$, while terms $\frac{1}{2} \Omega x_{2} x_{5}, \frac{1}{2} \Omega x_{1} x_{5}, \frac{1}{2} \Omega x_{4} x_{5}$ and $\frac{1}{2} \Omega x_{3} x_{5}$ will be added to lines one to four in the expression for $\mathbf{F}$. Note that the dependence of $\mathbf{F}$ on terms containing $x_{5}$ is of second (not of third) order. Moreover, since the time

\footnotetext{
${ }^{5}$ One may note that the matrix $\mathbf{A}$ is non-invertable, since it has two eigenvalues at zero, and worry whether the fact that $\mathbf{A} \mathbf{s}=\mathbf{q}$ is of third order in $(u, v)$ really implies that $\mathbf{s}$ is also of third order (since one cannot simply take $\mathbf{s}=\mathbf{A}^{-1} \mathbf{q}$ ). There is no problem, however, since $\mathbf{s}$ is an element of the stable eigenplane and, because of the invariant nature of the eigenplanes, so is $\mathbf{A s}=\mathbf{q}$. We can therefore write $\mathbf{s}=\mathbf{A}^{\text {inv }} \mathbf{q}$, where we can view $\mathbf{A}^{\text {inv }}$ as the inverse of $\mathbf{A}$ on the eigenplane $\Psi^{\text {s. }}$.
} 
derivative of $x_{5}$ is identically zero, matrix $\mathbf{A}$ will get an additional zero-valued fifth row and column, and $\mathbf{F}$ will get a zero-valued fifth line. This yields

$$
\mathbf{A}=\frac{\Omega}{2}\left[\begin{array}{ccccc}
-\frac{\gamma}{\Omega} & A_{c}+\frac{1}{2}-\frac{2 \omega_{1}^{2}}{\Omega^{2}} & 0 & 0 & 0 \\
A_{c}-\frac{1}{2}+\frac{2 \omega_{1}^{2}}{\Omega^{2}} & -\frac{\gamma}{\Omega} & 0 & 0 & 0 \\
0 & 0 & -\frac{\gamma}{\Omega} & A_{c}+\frac{1}{2}-\frac{2 \omega_{2}^{2}}{\Omega^{2}} & 0 \\
0 & 0 & A_{c}-\frac{1}{2}+\frac{2 \omega_{2}^{2}}{\Omega^{2}} & -\frac{\gamma}{\Omega} & 0 \\
0 & 0 & 0 & 0 & 0
\end{array}\right]
$$

$$
\mathbf{F}(\mathbf{x})=\frac{\omega_{1}^{2}}{32 \Omega}\left(\begin{array}{c}
\left(x_{1}^{2}+x_{2}^{2}\right) x_{2}+x_{2} x_{3}^{2}+3 x_{2} x_{4}^{2}+2 x_{1} x_{3} x_{4}+\frac{16 \Omega^{2}}{\omega_{1}^{2}} x_{2} x_{5} \\
-\left(x_{1}^{2}+x_{2}^{2}\right) x_{1}-x_{1} x_{4}^{2}-3 x_{1} x_{3}^{2}-2 x_{2} x_{3} x_{4}+\frac{16 \Omega^{2}}{\omega_{1}^{2}} x_{1} x_{5} \\
\left(1-\frac{48 L}{\Omega^{2}}\right)\left(x_{3}^{2}+x_{4}^{2}\right) x_{4}+x_{1}^{2} x_{4}+3 x_{2}^{2} x_{4}+2 x_{1} x_{2} x_{3}+\frac{16 \Omega^{2}}{\omega_{1}^{2}} x_{4} x_{5} \\
-\left(1-\frac{48 L}{\Omega^{2}}\right)\left(x_{3}^{2}+x_{4}^{2}\right) x_{4}-x_{2}^{2} x_{3}-3 x_{1}^{2} x_{3}-2 x_{1} x_{2} x_{4}+\frac{16 \Omega^{2}}{\omega_{1}^{2}} x_{3} x_{5} \\
0
\end{array}\right) .
$$

Matrix A will thus have three critical eigenvalues at $\mathbf{x}=\mathbf{0}$. Furthermore, there will be three critical eigenvectors $\mathbf{c}$, three critical 'transposed eigenvectors' $\mathbf{t}$, and $\Psi^{\mathrm{c}}$ will be parametrized by three scalars $u, v$, and $a$. The complete equations of motion can be written in a form similar to Eq. (B.18), with the exception that the function $\mathbf{F}$ will now appear as

$$
\mathbf{F}\left(u \mathbf{c}_{1}+v \mathbf{c}_{2}+a \mathbf{c}_{3}+\mathbf{s}\right)
$$

Strictly speaking, we should add a fourth equation governing the time dependence of $a$, but since we know $A-A_{c}$ to be time-independent, we can leave the number of equations at three. What then are the consequences of the inclusion of $x_{5}$ for the equations for $\dot{u}$ and $\dot{v}$ ? All the old terms (containing $u$ and $v$ only) remain the same, but we get extra terms containing $a$. To lowest order, we will find terms like $a u$ and $a v$ in the reduced equations of motion. Due to the tangent nature of the center manifold the expression for $\Delta: \mathbf{s}=\mathbf{s}(u, v, a)$ is, as before, at least of second order in $u, v$ and now $a$. Its new, $a$-dependent contribution to the equations for $\dot{u}$ and $\dot{v}$, via the argument of $\mathbf{F}$, will then be at least of order three so that, once again, we may skip the contribution of 
$\mathbf{s}$ to the reduced equations of motion. Keeping in mind that the equations for $\dot{u}$ and $\dot{v}$ must be odd in $u$ and $v$, respectively, we readily obtain

$$
\begin{aligned}
& \dot{u}=\mathbf{t}_{1} \cdot\left(\mathbf{F}_{u^{3}} u^{3}+\mathbf{F}_{u v^{2}} u v^{2}+\mathbf{F}_{u a} u a\right)+\text { h.o.t. } \\
& \dot{v}=\mathbf{t}_{2} \cdot\left(\mathbf{F}_{v^{3}} v^{3}+\mathbf{F}_{u^{2} v} u^{2} v+\mathbf{F}_{v a} v a\right)+\text { h.o.t. }
\end{aligned}
$$

for the $a$-dependent (and thus suitable for a bifurcation analysis) reduced equations of motion. The Taylor coefficients closely resemble the expressions in Eq. (B.21):

$$
\begin{aligned}
& \mathbf{F}_{u^{3}}=\left.\frac{1}{6} \frac{\partial^{3} \mathbf{F}\left(u \mathbf{c}_{1}+v \mathbf{c}_{2}+a \mathbf{c}_{3}\right)}{\partial u^{3}}\right|_{u, v, a=0}, \\
& \mathbf{F}_{u v^{2}}=\left.\frac{1}{2} \frac{\partial^{3} \mathbf{F}\left(u \mathbf{c}_{1}+v \mathbf{c}_{2}+a \mathbf{c}_{3}\right)}{\partial u \partial v^{2}}\right|_{u, v, a=0}, \\
& \mathbf{F}_{v^{3}}=\left.\frac{1}{6} \frac{\partial^{3} \mathbf{F}\left(u \mathbf{c}_{1}+v \mathbf{c}_{2}+a \mathbf{c}_{3}\right)}{\partial v^{3}}\right|_{u, v, a=0}, \\
& \mathbf{F}_{u^{2} v}=\left.\frac{1}{2} \frac{\partial^{3} \mathbf{F}\left(u \mathbf{c}_{1}+v \mathbf{c}_{2}+a \mathbf{c}_{3}\right)}{\partial u^{2} \partial v}\right|_{u, v, a=0}, \\
& \mathbf{F}_{u a}=\left.\frac{\partial^{2} \mathbf{F}\left(u \mathbf{c}_{1}+v \mathbf{c}_{2}+a \mathbf{c}_{3}\right)}{\partial u \partial a}\right|_{u, v, a=0}, \\
& \mathbf{F}_{v a}=\left.\frac{\partial^{2} \mathbf{F}\left(u \mathbf{c}_{1}+v \mathbf{c}_{2}+a \mathbf{c}_{3}\right)}{\partial v \partial a}\right|_{u, v, a=0},
\end{aligned}
$$

and can be calculated directly.

\section{B.4. Step three: normal form analysis}

In the previous steps we have successfully removed the explicit time dependence and all nonessential spatial dimensions from the equations of motion, and we are left with a two-dimensional problem governing the essence of the dynamics for values of the driving amplitude $A$ around the primary mode interaction point (for small values of $K$ ). The bifurcation diagrams we present in this subsection thus display the sequence of events when we follow, in contrast to our normal practice, a vertical path in the $(A, \Omega)$-plane close to the intersection point of the two resonance tongues.

The averaging has obviously removed the $Z_{2}(\mathrm{~T})$ from the symmetry group $\boldsymbol{Z}_{2}(\mathbf{R}) \times \boldsymbol{Z}_{2}(\mathrm{E}) \times \boldsymbol{Z}_{2}(\mathrm{~T})$ of the full equations of motion; the final form, Eq. (B.24), still 
has $\boldsymbol{Z}_{\mathbf{2}}(\mathbf{R}) \times \boldsymbol{Z}_{\mathbf{2}}(\mathbf{E})$ symmetry. Golubitsky et al. [16] state that our bifurcation problem g, defined as

$$
\left(\begin{array}{c}
\dot{u} \\
\dot{v}
\end{array}\right)=\mathbf{g}(u, v, a) \equiv\left(\begin{array}{c}
\mathbf{t}_{1} \cdot\left(\mathbf{F}_{u^{3}} u^{3}+\mathbf{F}_{u v^{2}} u v^{2}+\mathbf{F}_{u a} u a\right)+\text { h.o.t. } \\
\mathbf{t}_{2} \cdot\left(\mathbf{F}_{v^{3}} v^{3}+\mathbf{F}_{u^{2} v} u^{2} v+\mathbf{F}_{v a} v a\right)+\text { h.o.t. }
\end{array}\right)=\left(\begin{array}{l}
0 \\
0
\end{array}\right)
$$

is $\left(\boldsymbol{Z}_{2} \times \boldsymbol{Z}_{2}\right)$-equivalent to its normal form $\mathbf{h}$ :

$$
\mathbf{h}(u, v, a)=\left(\begin{array}{l}
\varepsilon_{1} u^{3}+m u v^{2}+\varepsilon_{2} a u \\
\varepsilon_{3} v^{3}+n u^{2} v+\varepsilon_{4} a v
\end{array}\right)=\left(\begin{array}{l}
0 \\
0
\end{array}\right)
$$

where

$$
\begin{aligned}
& \varepsilon_{1}=\operatorname{sgn}\left(\mathbf{t}_{1} \cdot \mathbf{F}_{u^{3}}\right), \\
& \varepsilon_{2}=\operatorname{sgn}\left(\mathbf{t}_{1} \cdot \mathbf{F}_{u a}\right), \\
& m=\left|\frac{\left(\mathbf{t}_{2} \cdot \mathbf{F}_{v a}\right)}{\left(\mathbf{t}_{2} \cdot \mathbf{F}_{v^{3}}\right)\left(\mathbf{t}_{1} \cdot \mathbf{F}_{u a}\right)}\right|\left(\mathbf{t}_{1} \cdot \mathbf{F}_{u v^{2}}\right), \\
& \varepsilon_{3}=\operatorname{sgn}\left(\mathbf{t}_{2} \cdot \mathbf{F}_{v^{3}}\right), \\
& \varepsilon_{4}=\operatorname{sgn}\left(\mathbf{t}_{2} \cdot \mathbf{F}_{v a}\right), \\
& n=\left|\frac{\left(\mathbf{t}_{1} \cdot \mathbf{F}_{u a}\right)}{\left(\mathbf{t}_{1} \cdot \mathbf{F}_{u^{3}}\right)\left(\mathbf{t}_{2} \cdot \mathbf{F}_{v a}\right)}\right|\left(\mathbf{t}_{2} \cdot \mathbf{F}_{u^{2} v}\right),
\end{aligned}
$$

provided the following nondegeneracy conditions are fulfilled:

$$
m \neq \varepsilon_{2} \varepsilon_{3} \varepsilon_{4}, \quad n \neq \varepsilon_{1} \varepsilon_{2} \varepsilon_{4}, \quad m n \neq \varepsilon_{1} \varepsilon_{3} .
$$

In words, this means that the bifurcational structure of $\mathbf{g}$ and $\mathbf{h}$ are qualitatively the same (also with respect to the symmetry characteristics), providing the conditions Eq. (B.32) are met.

Now, the inclusion of extra parameters in a bifurcation problem can cause the bifurcations to unfold. We have seen an example in the main text: the inclusion of the dissipation parameter $\gamma$ causes certain symmetry-breaking bifurcations to unfold (cf. Fig. 17 in the main text). It turns out that most bifurcation problems (and their normals forms) can unfold in only a finite number of ways and that one needs only a certain finite number of additional parameters to describe these; this number is called the codimension of the problem. ${ }^{6}$ We call $\tilde{\mathbf{h}}$ a universal unfolding of $\mathbf{h}$ if $\tilde{\mathbf{h}}$ describes all qualitatively different unfoldings of $\mathbf{h}$ and contains the minimum number of additional parameters; $\tilde{\mathbf{h}}$ reduces to $\mathbf{h}$ if all additional parameters are

\footnotetext{
${ }^{6}$ Some authors choose the codimension to include the bifurcation parameter $a$ of the original problem, which means that their codimension is (by definition) always 1 higher than ours.
} 
set to zero. The universal unfolding of the normal form given by Eq. (B.29) is known to be [16]

$$
\tilde{\mathbf{h}}(u, v, a, \tilde{m}, \tilde{n}, \sigma)=\left(\begin{array}{c}
\varepsilon_{1} u^{3}+\tilde{m} u v^{2}+\varepsilon_{2} a u \\
\varepsilon_{3} v^{3}+\tilde{n} u^{2} v+\varepsilon_{4}(a-\sigma) v
\end{array}\right),
$$

where $(\tilde{m}, \tilde{n}, \sigma)$ varies around $(m, n, 0)$.

The time has now come to calculate the values of the coefficients $\varepsilon_{1}, \varepsilon_{2}, \varepsilon_{3}, \varepsilon_{4}, m$ and $n$ using Eq. (B.30) and our knowledge of the (five-dimensional) matrix A(Eq. (B.23)) and the nonlinear function $\mathrm{F}(\mathrm{Eq}$. (B.24)). One finds

$$
\varepsilon_{1}=-1, \quad \varepsilon_{2}=+1, \quad \varepsilon_{4}=+1, \quad m=-\left|\frac{\omega_{1}^{2}}{\omega_{1}^{2}-48 L}\right|, \quad n=+1,
$$

and for $\varepsilon_{3}$ :

$$
\begin{aligned}
& \varepsilon_{3}=+1 \quad \text { if } L<\frac{\omega_{1}^{2}}{48}, \\
& \varepsilon_{3}=-1 \quad \text { if } L>\frac{\omega_{1}^{2}}{48} .
\end{aligned}
$$

Two things are immediately clear from Eq. (B.34) and the nondegeneracy conditions, Eq. (B.32). Firstly, we see that the bifurcation problem is degenerate when the pendulums are coupled by a linear spring, i.e., for $L=0$ (since then we have $m=-1$ and $\varepsilon_{3}=+1$, so $m n=\varepsilon_{1} \varepsilon_{3}$ ). In that case the above derivation of the normal form and the universal unfolding fails; in practice, this means that the bifurcation lines where MP is born from $1 \alpha$ and $2 \beta$ (that is, line $\mathbf{R}_{\mathbf{I}, \text { left }}$ and line $\mathbf{G}_{\mathbf{I}}$ in Fig. 21 of Ref. [2]) run very close together, as was already mentioned in [2]. (The results of this appendix will, of course, not be in exact quantitative agreement with the results of the main text, since the derivations presented here are valid only if the linear coupling parameter $K$ is small enough). Secondly, we see from Eq. (B.33) that $L=\omega_{1}^{2} / 48$ is evidently some critical value, for which we may expect the bifurcational structure to change qualitatively. It turns out that, for $L<\omega_{1}^{2} / 48$, one of the two normal motions (the $2 \beta$-motion) is born subcritically from the trivial motion whereas it is born supercritically for $L>\omega_{1}^{2} / 48$. Indeed, this corresponds to the $2 \beta$-motion being born 'soft' for small $L$ and 'hard' for sufficiently large values of $L$ (see main text).

A comprehensive listing of all possible unfoldings of the bifurcation problem defined by Eq. (B.28) can be found in Golubitsky et al. [16] and Langford and Iooss [17]. Here we reproduce the three cases that are relevant for us. In Fig. B.2a and Fig. B.2b the unfoldings are sketched for $L<0$, in Fig. B.3a and Fig. B.3b for $0<L<\omega_{1}^{2} / 48$, and Fig. B.4a and Fig. B.4b gives the sequence of events for $L>\omega_{1}^{2} / 48$. In these figures the diagrams for $\sigma>0$ represent the situation for increasing driving amplitude left of the primary mode interaction point, whereas $\sigma<0$ corresponds to the situation right of the primary mode interaction point, as indicated in Fig. B.2c., Fig. B.3c and Fig. B.4c. Furthermore, $u$ is associated with $1 \alpha$ and $v$ with $2 \beta$. 


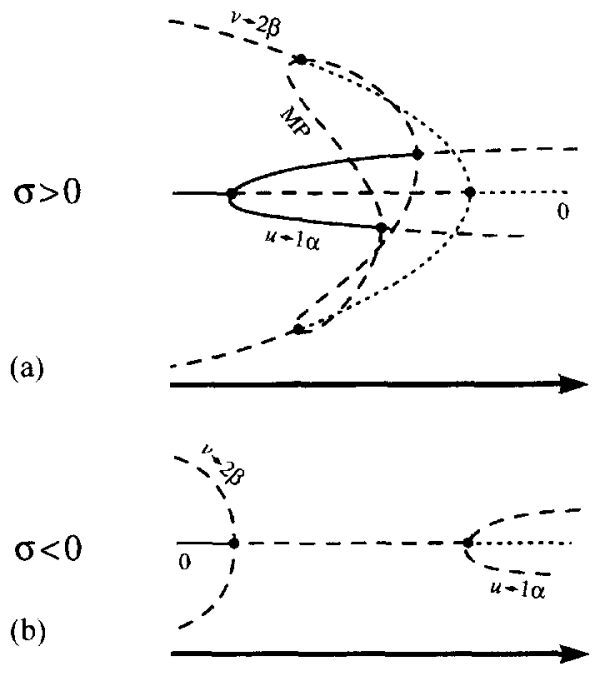

(c)

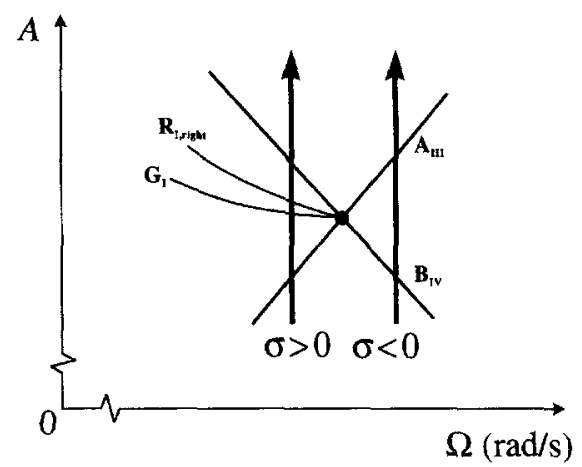

Fig. B.2. The bifurcational structure close to the primary mode interaction point for $L<0$, with $\Omega$ fixed and $A$ increasing. (a) For $\sigma>0$ (to the left of the mode interaction point), (b) for $\sigma<0$ (to the right of the mode interaction point), and (c) the corresponding bifurcation lines in the $(A, \Omega)$-plane.

For $L<0$ (Fig. B.2) we see that line $\mathbf{G}_{\mathrm{I}}$ lies below line $\mathbf{R}_{\mathbf{1} \text { left }}$, while for $0<L<\omega_{1}^{2} / 48$ (Fig. B.3) the situation is reversed. This means that for $L=0$ the two lines coincide (close to the primary mode interaction point), confirming our earlier observation about the degeneracy for this value of $L$. Indeed, linear coupling is convenient as far as the equations of motion (and most of the dynamics, as described in Refs. [1,2]) are concerned, but the intricacies of mode interaction are far more transparent when a nonlinear term is added to the coupling.

Going from $0<L<\omega_{1}^{2} / 48$ (Fig. B.3) to $L>\omega_{1}^{2} / 48$ (Fig. B.4) we see that line $\mathbf{G}_{1}$ rises above line $\mathbf{B}_{\mathrm{IV}}$, confirming the fact that the $2 \beta$-motion is born 'hard' if the nonlinear term in the coupling is sufficiently large. 

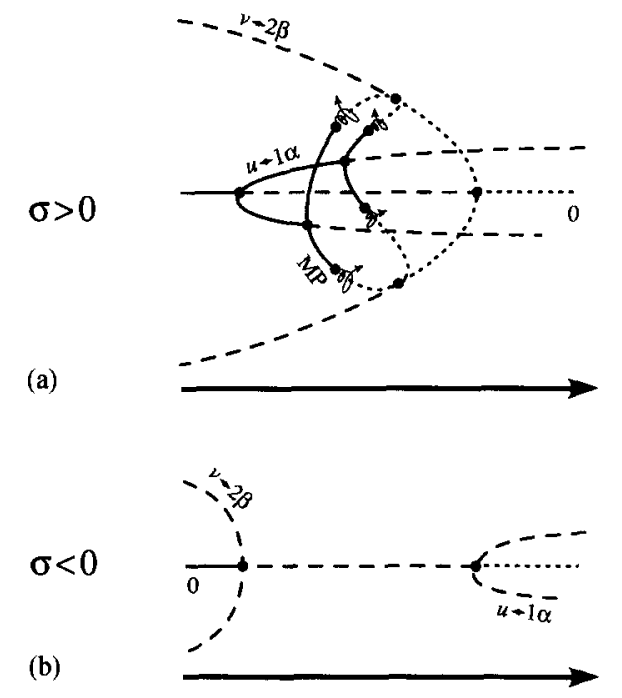

(c)

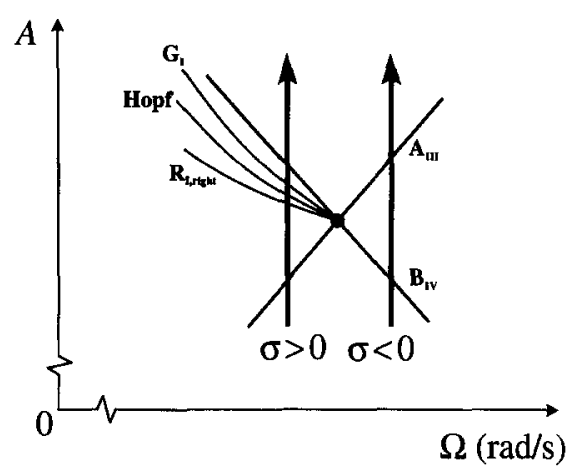

Fig. B.3. The same as Fig. B.2 but now for $0<L<\omega_{1}^{2} / 48$.

There is one final remark that should be made, concerning the stability of the solutions depicted in Fig. B.2-Fig. B.4. Although there is a one-to-one correspondence between the solutions of the bifurcation problem $\mathbf{g}$ and its normal form $\mathbf{h}$ (since the two are $\left(Z_{2} \times Z_{2}\right)$-equivalent $)$, the stability of the mixed mode solutions need not be the same. We have depicted the simplest scenarios, i.e., the ones that contain the minimum number of bifurcations, but these are not always the ones observed in practice. For example, in Fig. B.4a the mixed motion is depicted to be stable everywhere, while in the two-pendulum system we actually found two Hopf bifurcations rendering it unstable during part of its existence. The only thing guaranteed by the present analysis is that the MP-motion in Fig. B.4a is born stable at both ends (at $\mathbf{R}_{\mathrm{I} \text {, right }}$ as well as at $\mathbf{G}_{\mathrm{I}}$ ), because it takes over the stability properties of the respective mother orbits ( $1 \alpha$ and $2 \beta)$; it does not tell us what happens in between. 

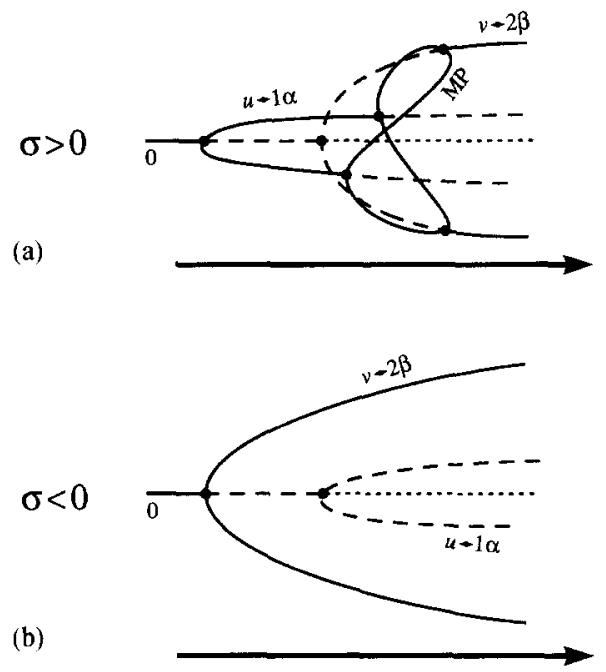

(b)

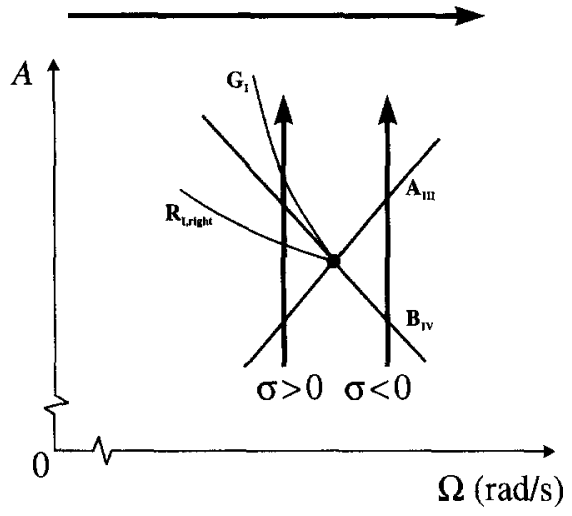

Fig. B.4. The same as Fig. B.2 but now for $L>\omega_{1}^{2} / 48$.

Whether the mixed motion follows the depicted (simplest) scenario, or another one with additional bifurcations, is determined by terms in $\mathbf{g}$ of fifth and higher order. We shall, however, not pursue the matter here but instead rely on the numerical methods used in the main text.

\section{References}

[1] E.J. Banning, J.P. van der Weele, Mode competition in a system of two parametrically driven pendulums; the Hamiltonian case, Physica A 220 (1995) 485-533

[2] E.J. Banning, J.P. van der Weele, J.C. Ross, M.M. Kettenis, E. de Kleine, Mode competition in a system of two parametrically driven pendulums; the dissipative case, Physica A 245 (1997) 11-48, this issue.

[3] S. Ciliberto, J.P. Gollub, Pattern competition leads to chaos, Phys. Rev. Lett. 52 (1984) $922-925$.

[4] S. Ciliberto, J.P. Gollub, Chaotic mode competition in parametrically forced surface waves, J. Fluid Mech. 158 (1985) 381-398. 
[5] H. Ayanle, A. Bernoff, S. Lichter, Spanwise modal competition in cross-waves, Physica D 43 (1990) $87-104$.

[6] L. Shemer, S. Lichter, The mode number dependence of neutral stability of cross-waves, Experiments in Fluids 9 (1990) 148-152.

[7] W.B. Underhill, S. Lichter, A.J. Bernoff, Modulated, frequency-locked, and chaotic cross-waves, J. Fluid Mech. 225 (1991) 371-394.

[8] A.C. Skeldon, T. Mullin, Mode interaction in a double pendulum, Phys. Lett. A 166 (1992) 224-229.

[9] A.C. Skeldon, Dynamics of a Parametrically excited double pendulum, Physica D 75 (1994) 541-558.

[10] E.J. Banning, J.P. van der Weele, M.M. Kettenis, Mode competition in a system of two parametrically driven pendulums; the role of symmetry, Physica A, in press (1997).

[11] J.E. Howard, R.S. MacKay, Linear stability of symplectic maps, J. Math. Phys. 28 (1987) 1036-1051.

[12] Th. Zeegers, On the existence of infinite period-doubling sequences in a class of $4 \mathrm{D}$ semi-symplectic mappings, J. Phys. A: Math. Gen. 24 (1991) 2287-2314.

[13] S. Wiggins, Introduction to Applied Nonlinear Dynamical Systems and Chaos, Applied Mathematics, Springer Verlag, New York, 1990.

[14] J. Guckenheimer, P. Holmes, Nonlinear Oscillations, Dynamical Systems and Bifurcations of Vector Fields, Applied Mathematical Sciences, vol. 42, Springer Verlag, New York, 1983.

[15] Y.A. Kuznetsov, Elements of Applied Bifurcation Theory, Applied Mathematical Sciences, vol. 112, Springer Verlag, New York, 1991.

[16] M. Golubitsky, D.G. Schaeffer, Singularities and Groups in Bifurcation Theory. vol. 1, Applied Mathematical Sciences, vol. 51, Springer Verlag, New York, 1985.

[17] W.F. Langford, G. Iooss, Interactions of Hopf and pitchfork bifurcations, in: H.D. Mittelmann, H. Weber (eds.), Bifurcation Problems and their Numerical Solution, International Series of Numerical Mathematics, vol. 54, Birkhäuser Verlag, Basel, 1980, pp. 103-134. 